\title{
Charge Order Breaks Magnetic Symmetry in Molecular Quantum Spin Chains
}

\author{
M. Dressel, M. Dumm, T. Knoblauch, B. Köhler, B. Salameh, and S. Yasin \\ 1. Physikalisches Institut, Universität Stuttgart, Pfaffenwaldring 57, 70550 Stuttgart, Germany \\ Correspondence should be addressed to M. Dressel, dressel@pil.physik.uni-stuttgart.de \\ Received 2 September 2012; Accepted 11 October 2012 \\ Academic Editor: Fabrizio Carbone \\ Copyright () 2012 M. Dressel et al. This is an open access article distributed under the Creative Commons Attribution License, \\ which permits unrestricted use, distribution, and reproduction in any medium, provided the original work is properly cited.
}

Charge order affects most of the electronic properties but is believed not to alter the spin arrangement since the magnetic susceptibility remains unchanged. We present electron-spin-resonance experiments on quasi-one-dimensional (TMTTF) ${ }_{2} X$ salts $\left(X=\mathrm{PF}_{6}, \mathrm{AsF}_{6}\right.$, and $\left.\mathrm{SbF}_{6}\right)$, which reveal that the magnetic properties are modified below $T_{\mathrm{CO}}$ when electronic ferroelectricity sets in. The coupling of anions and organic molecules rotates the g-tensor out of the molecular plane creating magnetically nonequivalent sites on neighboring chains at domain walls. Due to anisotropic Zeeman interaction a novel magnetic interaction mechanism in the charge-ordered state is observed as a doubling of the rotational periodicity of $\Delta H$.

\section{Introduction}

Charge disproportionation is a common ordering mechanism in correlated electron systems that is intensively investigated in transition-metal oxides as well as organic compounds $[1,2]$ because it is recognized to influence charge, lattice, and magnetic degrees of freedom. For static charge order (CO) the electrons are (partially) localized resulting in an insulating state, most evident in manganites or at the $1 / 8$ anomaly observed in cuprates [3]. Fluctuating stripes, however, may be relevant for high-temperature superconductivity [4], but also in organic systems evidence accumulates that charge fluctuations mediate superconductivity [5-7].

As far as the magnetic properties are concerned, the situation is even more puzzling. CO can induce ferroelectricity when the inversion symmetry is broken, as suggested (and also disputed) for some mixed-valence oxides [9, 10], magnetite [11-15], manganites $[16,17]$, or nickelates [18, 19]. For perovskites, however, there seems to be a mutual exclusion of magnetism and ferroelectricity [20-22], albeit the field of multiferroics drew enormous attention over the last years [23-25]. About ten years ago, electronic ferroelectricity was discovered in quasi-one-dimensional organic compounds (TMTTF) ${ }_{2} X$ (where TMTTF stands for tetramethyltetrathiafulvalene and $X$ denotes a monovalent anion such as $\mathrm{PF}_{6}, \mathrm{AsF}_{6}, \mathrm{SbF}_{6}, \mathrm{Br}$ ) around 50-160 K [26]. Xray investigations could not identify any superstructure, on this account it is known as a "structureless" transition [27].

In these strongly correlated electron systems, nearestneighbor Coulomb repulsion causes CO upon cooling below $T_{\mathrm{CO}}$ characterized by a charge gap in the transport properties [28]. From the magnetic point of view, these are $S=1 / 2$ antiferromagnetic chains with some ordering at low temperatures $[29,30]$. Surprisingly, by now no indications of the $\mathrm{CO}$ transition could be observed in the magnetic properties, such as spin susceptibility (cf. Figure 1). Here we, report comprehensive electron spin resonance (ESR) investigations that reveal a novel magnetic interaction mechanism caused by charge disproportionation; we observe first evidence that charge order breaks the symmetry of the magnetic degree of freedom in these quantum spin chains.

\section{Results and Analysis}

Single crystals of (TMTTF $)_{2} X$ with octahedral anions $X=$ $\mathrm{PF}_{6}, \mathrm{AsF}_{6}$, and $\mathrm{SbF}_{6}$, are grown electrochemically. The planar 


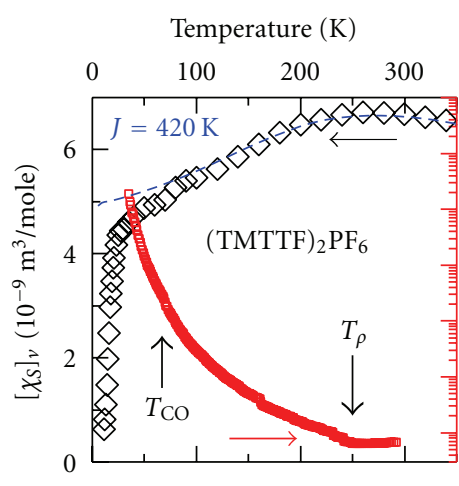

(a)

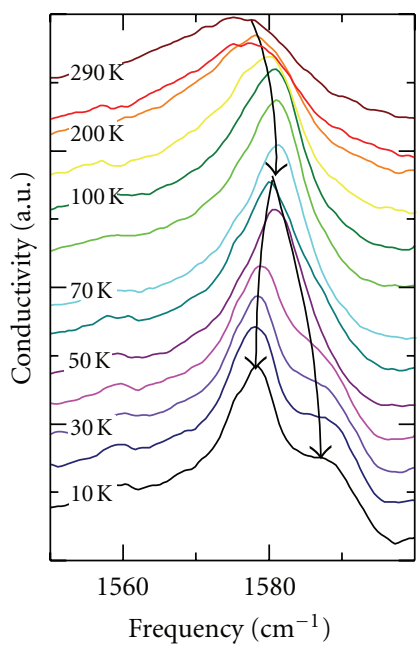

(d)

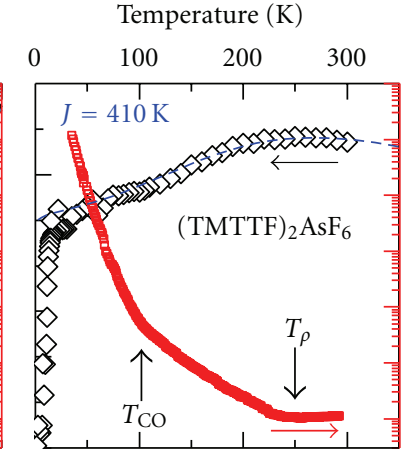

(b)

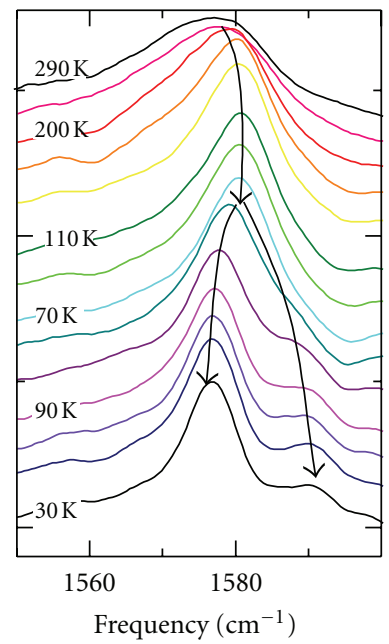

(e)

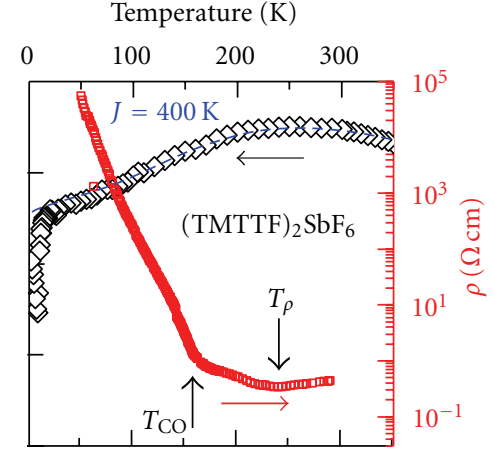

(c)

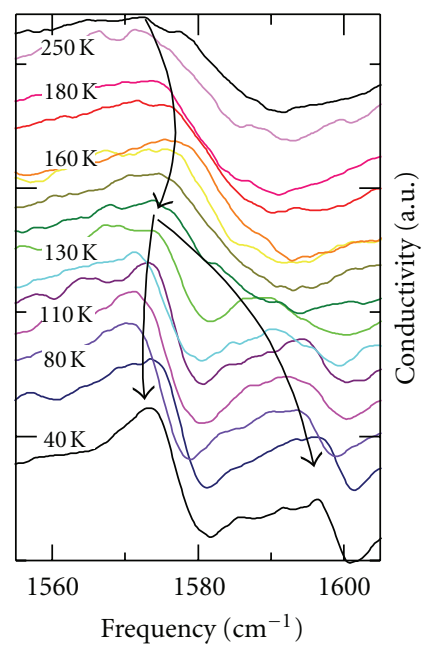

(f)

Figure 1: Electronic, magnetic, and optical characterization of the three compounds $(\mathrm{TMTTF})_{2} \mathrm{PF}_{6},(\mathrm{TMTTF})_{2} \mathrm{AsF}_{6}, \mathrm{and}(\mathrm{TMTTF})_{2} \mathrm{SbF}_{6}$. (a)-(c) The open symbols correspond to the spin susceptibility $\left(\chi_{s}\right)_{V}(T)$ along the $a$ direction evaluated at constant volume (left axis). The dashed blue lines correspond to fits using the model [8] of a Heisenberg chain with $J$ as indicated. For $(\mathrm{TMTTF})_{2} \mathrm{PF}_{6}$ and $(\mathrm{TMTTF})_{2} \mathrm{AsF}_{6}$, $\left(\chi_{s}\right)_{V}(T)$ drops exponentially at $T_{\mathrm{SP}}=19$ and $13 \mathrm{~K}$, respectively, indicating a transition to a nonmagnetic spin-Peierls ground state. In contrast, (TMTTF) $)_{2} \mathrm{SbF}_{6}$ orders antiferromagnetically at $T_{N}=8 \mathrm{~K}$. The solid red dots show the temperature-dependent dc resistivity $\rho(T)$ measured along the $a$-axis (right scale). The solid arrows indicate the charge localization at $T_{\rho}$ and the charge-order transition $T_{\mathrm{CO}}(\mathrm{d})-(\mathrm{f})$ Temperature development of the asymmetric vibration of the TMTTF molecule involving the $\mathrm{C}=\mathrm{C}$ bonds obtained with light polarized perpendicular to the $a b$ plane. For $T<T_{\mathrm{CO}}$ the mode splits due to charge imbalance.

TMTTF molecules stack in a slight zig-zag fashion normal to the molecular plane; this $a$-axis is the best-conducting direction. The stacks are arranged next to each other with some minor interaction in $b$ direction and separated in the $c$ direction by the anions. Since one electron is transferred to each anion, the organic molecules are left with half a hole, on average. Triggered by the $A_{2} B$ stoichiometry, the TMTTF molecules form dimers along the $a$-axis; a fact that enhances the charge localization observed as a broad minimum in resistivity at $T_{\rho} \approx 250 \mathrm{~K}$ [31-35]. In Figures $1(\mathrm{a})-1(\mathrm{c}), \rho(T)$ is plotted for the three compounds under consideration [28].

In addition, basically all TMTTF salts develop charge imbalance for $T<T_{\mathrm{CO}}$, resulting in charge-rich $(0.5 e+\delta)$ and charge-poor molecules $(0.5 e-\delta)$ as first proven by nuclear magnetic resonance $[36,37]$. The charge disproportionation can be best visualized by the splitting of the intramolecular vibration $\nu_{28}\left(B_{1 u}\right)$ plotted in Figures $1(\mathrm{~d})$ 1(f) [38-40]. The results are summarized in Table 1; for further details, see [41].

Charge order causes an additional gap to open in the density of states at $T_{\mathrm{CO}}$, leading to a kink in the electrical resistivity $\rho(T)$ indicated by arrows in Figures $1(\mathrm{a})-1(\mathrm{c})$; as the temperature decreases it evolves in a mean-field fashion to $\Delta_{\mathrm{CO}}(T \rightarrow 0) \approx 745 \mathrm{~K}$, for the example of $(\mathrm{TMTTF})_{2} \mathrm{SbF}_{6}$ [28].

Although the effect of charge ordering on the spin degree of freedom has attracted considerable attention from the theoretical side [42], it is not completely clear how the spin and orbital magnetic moments behave in the charge-order state and in the metallic state under the influence of charge fluctuations. Naively, one would expect that a phase transition, which has such a severe effect on the electric transport, 
TABle 1: Transition temperatures for charge localization $T_{\rho}$, charge-order $T_{\mathrm{CO}}$, spin-Peierls transition $T_{\mathrm{SP}}$ and Néel order $T_{N}$ of different (TMTTF) ${ }_{2} X$ salts. $J$ denotes the antiferromagnetic exchange constant obtained from susceptibility measurements fitted according to [8], $2 \delta$ is the charge imbalance between two TMTTF molecules (for $T \rightarrow 0$ ) measured by vibrational spectroscopy and $\Delta_{\text {Co }}$ indicate the zerotemperature charge gap derived from dc transport along the $a$-direction. $\phi$ is the angle the g-tensor rotates around the molecular axis in the CO state determined at $T \approx T_{\mathrm{CO}} / 2$.

\begin{tabular}{lcccccccr}
\hline Compound & $\begin{array}{c}T_{\rho} \\
(\mathrm{K})\end{array}$ & $\begin{array}{c}T_{\mathrm{CO}} \\
(\mathrm{K})\end{array}$ & $\begin{array}{c}T_{\mathrm{SP}} \\
(\mathrm{K})\end{array}$ & $\begin{array}{c}T_{N} \\
(\mathrm{~K})\end{array}$ & $\begin{array}{c}J \\
(\mathrm{~K})\end{array}$ & $\begin{array}{c}2 \delta \\
(\mathrm{e})\end{array}$ & $\begin{array}{c}\Delta_{\mathrm{CO}} \\
(\mathrm{K})\end{array}$ & $\begin{array}{c}\phi \\
\left({ }^{\circ}\right)\end{array}$ \\
\hline$(\mathrm{TMTTF})_{2} \mathrm{PF}_{6}$ & 250 & 67 & 19 & - & 420 & 0.15 & small & 22 \\
$(\mathrm{TMTTF})_{2} \mathrm{AsF}_{6}$ & 250 & 102 & 13 & - & 410 & 0.21 & 310 & 22 \\
$(\mathrm{TMTTF})_{2} \mathrm{SbF}_{6}$ & 240 & 157 & - & 8 & 400 & 0.29 & 745 & 32 \\
\hline
\end{tabular}

evidences also in the spin susceptibility, albeit spin-charge separation is an issue for one-dimensional conductors [34, 35]. Despite numerous charge-ordered systems investigated over the years, no sign of the charge-order was revealed in the magnetic behavior. This is demonstrated in Figures 1(a)$1(\mathrm{c})$ which show $\chi_{s}(T)$ for various TMTTF salts $[29,30]$. The obtained coupling constants $J$ are listed in Table 1.

We have performed comprehensive multifrequency ESR investigations (X-, Q- and W-band) in the temperature range from 4 to $300 \mathrm{~K}$ at different orientations of the magnetic field and by rotating around the molecular axes (cf. Figure 6). In Figure 2 the linewidth $\Delta H(T)$ and the $g$-shift (from the free-electron value $\left.g_{e}=2.002319\right)$ are plotted as a function of temperature of (TMTTF $)_{2} \mathrm{SbF}_{6}$. The $g$-factor is determined by the $\pi$ electronic wavefunction on the TMTTF molecule, the anisotropy arises from spin-orbit coupling of the conduction electrons $[43,44]$; in other words the arrangement of the g-tensor can be solely deduced from the molecular symmetry. At room temperature the $g$-factor and linewidth are maximum when the external magnetic field is oriented along the molecular axis $\tilde{c}$, while $g$ and $\Delta H$ are smallest when measured normal to the TMTTF molecules ( $\tilde{a}$-axis which is along the stacks). No indications of the charge-order transition are observed in the $g$-shift; $\Delta g(T)$ is basically temperature independent except a small reduction of the in-plane anisotropy upon cooling. The intriguing observation is the clear anomaly in the linewidth at $T_{\mathrm{CO}}$ which can also be seen in [45]: the linear decrease in $\Delta H(T)$ becomes weaker indicating an additional interaction channel to develop for $T<T_{\mathrm{CO}}$. Basically, the same behavior at $T_{\mathrm{CO}}$ is observed for (TMTTF) ${ }_{2} \mathrm{AsF}_{6}$, while for (TMTTF) ${ }_{2} \mathrm{PF}_{6}$ the effect is smaller due to stronger coupling $J$ and less charge imbalance $2 \rho$, which coincide with the much lower transition temperature $T_{\mathrm{CO}}$ and almost vanishing charge gap $\Delta_{\mathrm{CO}}$, summarized in Table 1.

In order to gain more insight how charge order affects the spins, we performed detailed angular-dependent measurements of $\Delta H$ and $\Delta g$ at selected temperatures. While the rotational symmetry of the $\Delta g$ remains unchanged with temperature, $\Delta H$ exhibits a very unusual behavior as demonstrated in Figure 3. For $T>T_{\mathrm{CO}}$ the $g$-factor and the linewidth are dominated by spin-phonon scattering with an angular dependence [29]

$$
\Delta H_{\mathrm{sp}}(\theta)=\left[\Delta H_{\mathrm{sp}}^{2}(\widetilde{a}) \cos ^{2} \theta+\Delta H_{\mathrm{sp}}^{2}(\tilde{b}) \sin ^{2} \theta\right]^{1 / 2}
$$

in the $\tilde{a} \tilde{b}$ plane, for instance. Most remarkably, the periodicity doubles as the charge-ordered state is entered. In the CO state the orientational dependence of the linewidth can be fitted by the sum of (1) and (2):

$$
\Delta H_{\bmod }(\theta)=\Delta H_{\bmod }\left(45^{\circ}\right)|\sin \{2 \theta\}|,
$$

where $\Delta H_{\text {mod }}$ is the excess linewidth. The enhancement of the linewidth observed in the diagonal direction $\Delta H_{\mathrm{en}}=$ $\Delta H\left(45^{\circ}\right)-(1 / 2)[\Delta H(\tilde{a})+\Delta H(\tilde{b})]$ becomes more pronounced as the frequency $v$ increases, as depicted in Figure 4. The quadratic behavior

$$
\Delta H_{\mathrm{en}}(v)=A+B v^{2}
$$

strongly indicates anisotropic Zeeman interaction [46]. The same period doubling in $\Delta H(\theta)$ can be observed in our Xband experiments on (TMTTF) ${ }_{2} \mathrm{PF}_{6}$, although the effect is much weaker.

In the case of staggered fields, antisymmetric Dzyaloshinskii-Moriya interaction also causes a $v^{2}$ frequency dependence of the linewidth; however, leading to a $T^{-2}$ temperature dependence [47-49] in contrast to our findings. Note also that in those references Heisenberg spin chains with significant intrachain Dzyaloshinskii-Moriya interaction were considered. In the present case, the alternating contributions result from interaction between the chains, for which the iostropic exchange $J^{\prime}$ is already small. Thus the antisymmetric Dzyaloshinskii-Moriya exchange can be neglected.

From similar observations on inorganic linear-chain compounds, Pilawa derived a contribution of the anisotropic Zeeman interaction to the linewidth [50]

$$
\Delta H_{\mathrm{AZ}} \approx \frac{\mu_{B} H_{0}^{2}}{g_{e} k_{B}\left|J^{\prime}\right|} \sqrt{\frac{\pi}{8}}|\Delta g|^{2},
$$

where $J^{\prime}$ is the exchange interaction between two inequivalent magnetic sites on the neighboring chains; $\Delta g$ is the difference between their g-tensors. In our case the interchain exchange constant is $J^{\prime}=1.1 \mathrm{~K}$ [51]. Note, their Hamiltonian differs by a factor of 2 compared to the one used in [50]. With $B_{0}=33.6 \mathrm{kOe}$ for the W-band, we obtain $\Delta g=7.82 \times 10^{-3}$ and $16.78 \times 10^{-3}$ for $(\mathrm{TMTTF})_{2} \mathrm{AsF}_{6}$ and $(\mathrm{TMTTF})_{2} \mathrm{SbF}_{6}$, respectively. In the case of (TMTTF) ${ }_{2} \mathrm{PF}_{6}$ we performed only $\mathrm{X}$-band measurements which in general yield results similar to the Q-band; we estimate $\Delta g=7.45 \times 10^{-3}$. The fact that 


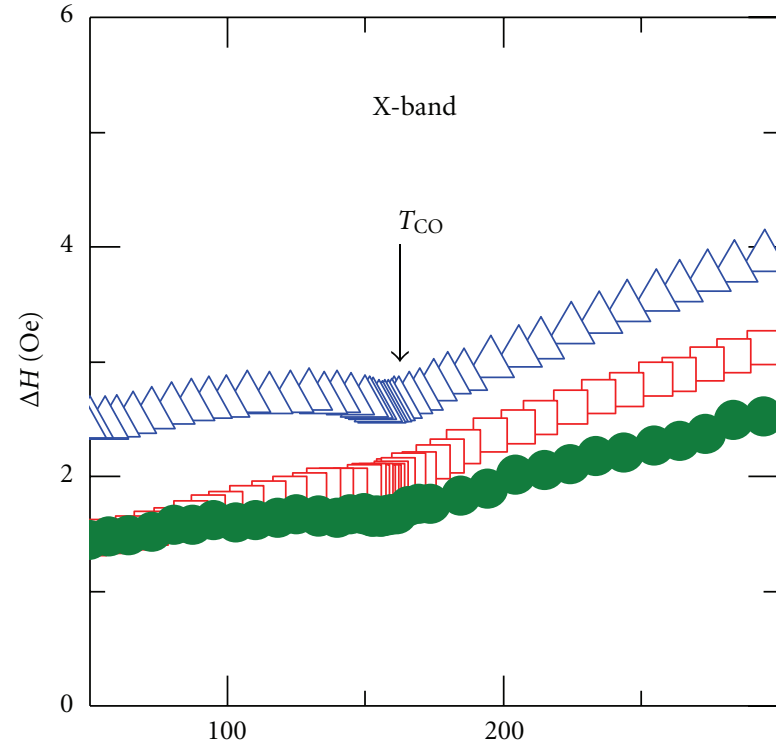

(a)

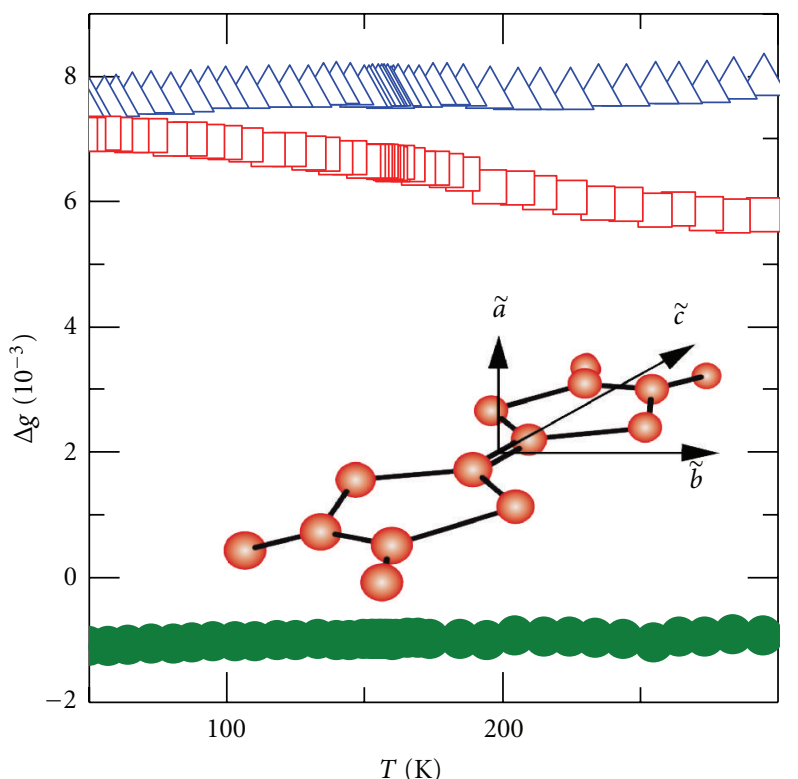

$\begin{array}{ll}\triangle \tilde{c} \text {-axis } \\ \square & \tilde{b} \text {-axis } \\ \tilde{a} \text {-axis }\end{array}$

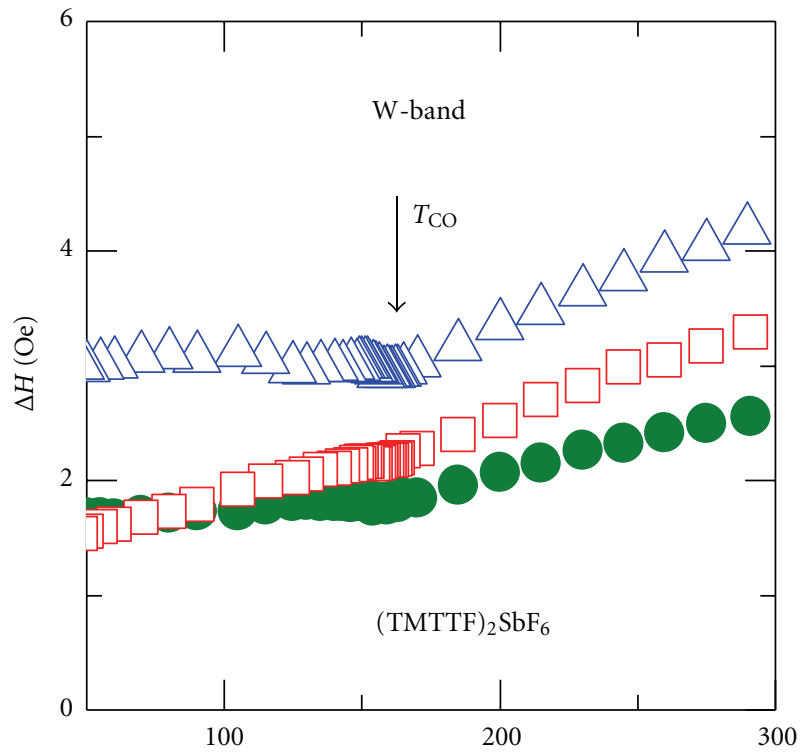

(c)

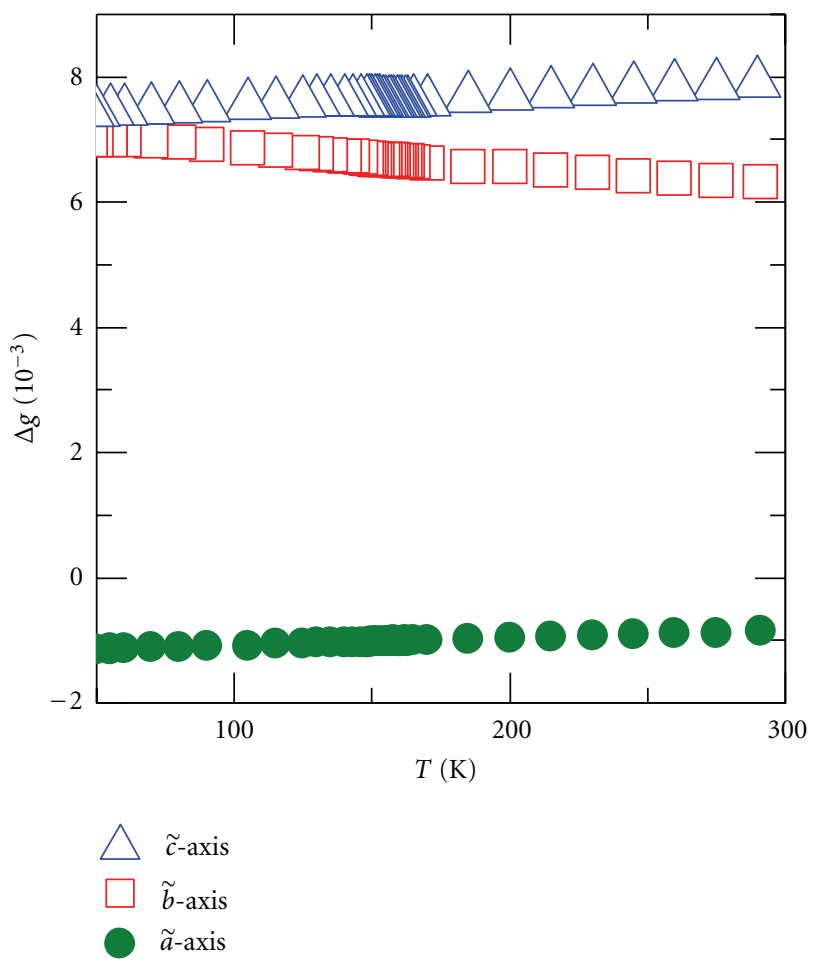

(d)

FIgURE 2: Temperature dependence of the linewidth $\Delta H$, and the change in the $g$-value for (TMTTF) $)_{2} \mathrm{SbF}_{6}$ measured by X- (left panels) and W-band (right panels) ESR along three orientations. The green solid dots correspond to the $\tilde{a}$-axis, the open red squares indicate the $\tilde{b}$ direction, and the blue triangles are measured along the $\tilde{c}$ direction. The inset exhibits the TMTTF molecule with its molecular axes.

we do not observe two distinct features but only a single Lorentzian line implies that the hopping rate between the nonequivalent sites is much larger than the splitting (strongcoupling limit). The difference in Larmor frequencies in the
W-band $\Delta v=(\Delta g / g) \times 95 \mathrm{GHz}$ corresponds to only $27 \mathrm{mK}$, which is much smaller than $J^{\prime}$.

The magnetically inequivalent sites have to be located on adjacent stacks; intrachain interaction is so strong that 


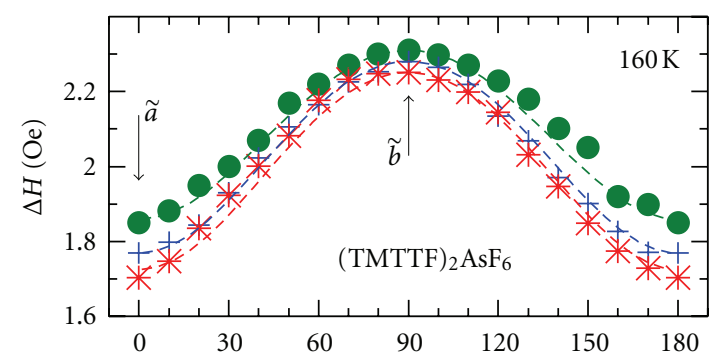

(a)

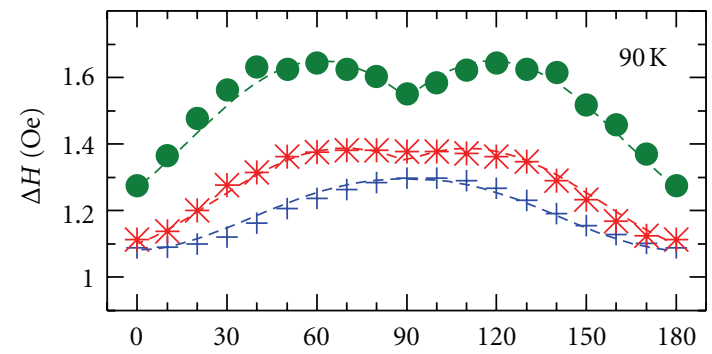

(c)

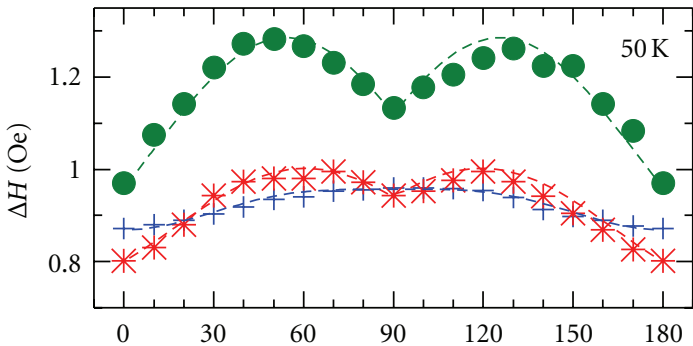

(e)

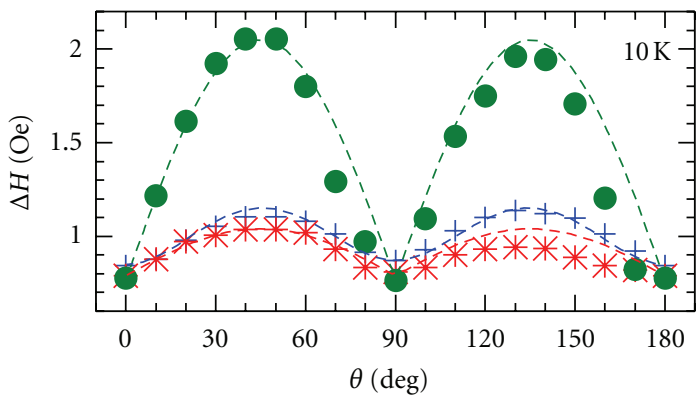

(g)

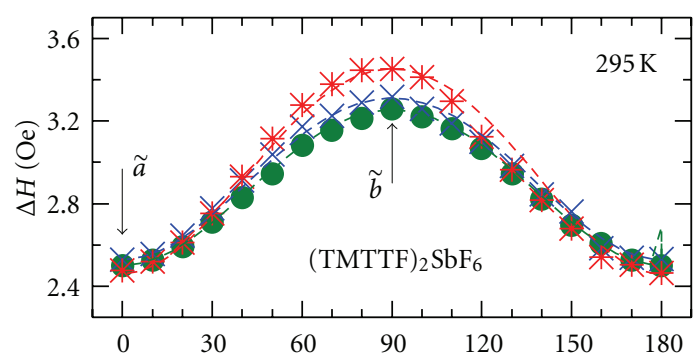

(b)

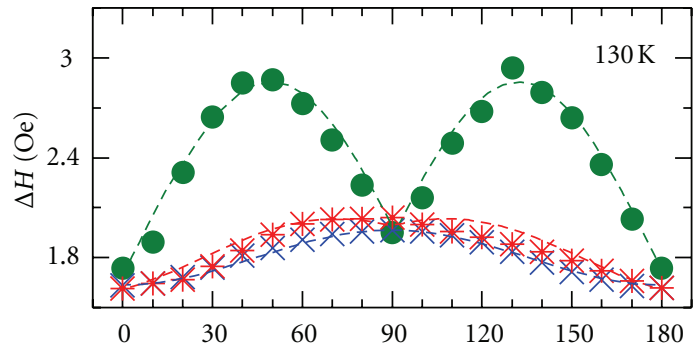

(d)

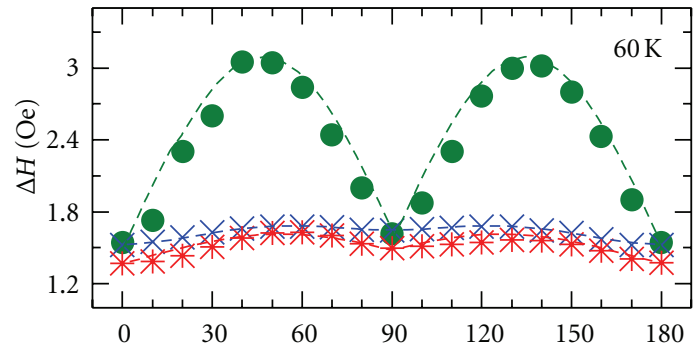

(f)

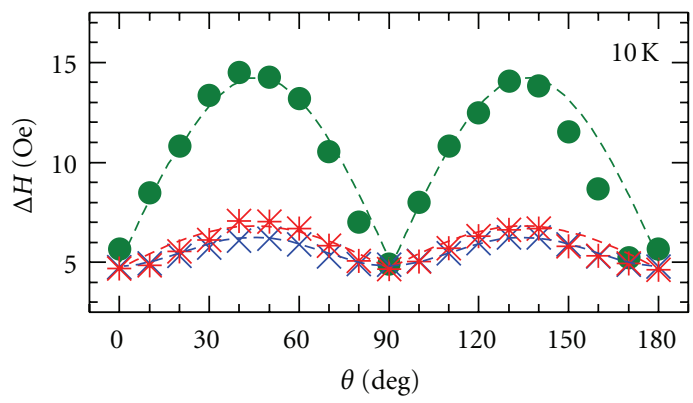

(h)

FIgURE 3: Angular dependence of the ESR linewidth within the $\tilde{a} \tilde{b}$ plane of (TMTTF) ${ }_{2} \mathrm{AsF}_{6}$ and (TMTTF) ${ }_{2} \mathrm{SbF}_{6}$ measured at different temperatures above and below the charge-order transition $\left(T_{\mathrm{CO}}=102\right.$ and $157 \mathrm{~K}$, resp.) using X-band (blue crosses), Q-band (red stars), and W-band (green dots) spectrometers. The lines represent fits of the linewidth using (1) and (2).

it would suppress the anisotropic Zeeman effect at these frequencies. From the fact that the maximum of $\Delta H$ occurs at $\theta=45^{\circ}$ and that $\Delta g$ is not zero, we conclude that the gtensors of the two sites do not coincide with the principle magnetic axes any more but are rotated around the $\tilde{c}$-axis by an angle $\phi$ in opposite directions as depicted in Figure 5; at temperature well below $T_{\mathrm{CO}}$ we obtain $\phi= \pm 22^{\circ}$ for $X=\mathrm{PF}_{6}, \mathrm{AsF}_{6}$ and $\pm 32^{\circ}$ for $(\mathrm{TMTTF})_{2} \mathrm{SbF}_{6}$, respectively Note that this effect is distinct from the gradual rotation of the g-tensor around the $\tilde{a}$-axis due to thermal contraction, reported by Furukawa et al. [44]. That rotation causes a reduction of the $g$-value anisotropy in the $\tilde{b} \tilde{c}$ plane as the temperature is reduced (Figure 2).

\section{Discussion}

The crucial question now is why $\mathrm{CO}$ triggers a rotation of the g-tensors. ESR experiments by Furukawa et al. [44] revealed that the Coulomb potential of the anions influences 


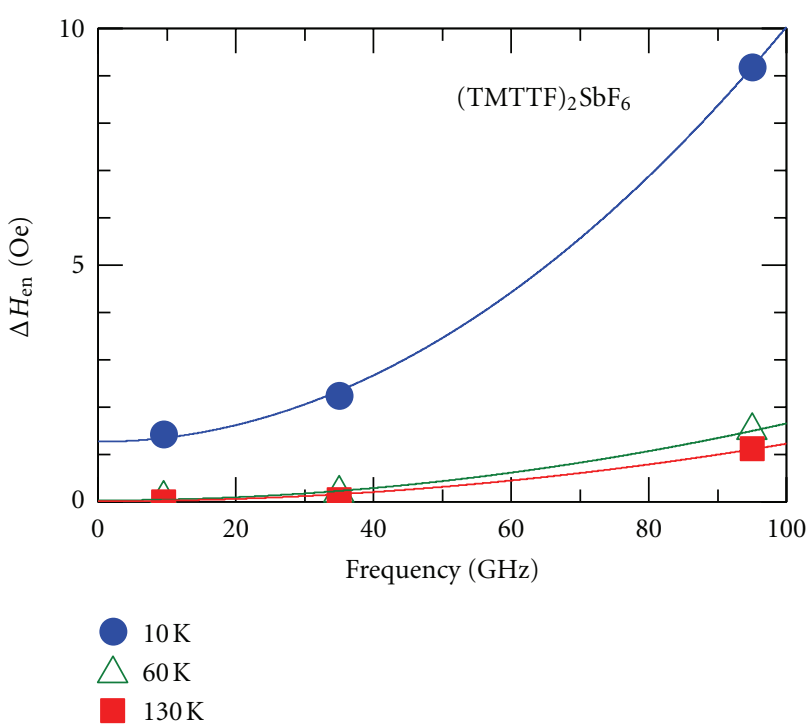

FIGURE 4: Frequency dependence of the enhanced ESR linewidth $\Delta H_{\mathrm{en}}$ of (TMTTF) $)_{2} \mathrm{SbF}_{6}$ measured in the $\tilde{a} \tilde{b}$ plane along the diagonal direction $\left(\theta=45^{\circ}\right)$. The data are shown at $T=10 \mathrm{~K}$ (blue dots), $60 \mathrm{~K}$ (green triangles), and $130 \mathrm{~K}$ (red squares). The lines are fits using (3).

the electronic wavefunctions on the TMTTF molecules. Depending on whether the adjacent molecules are charge rich or charge poor, this constitutes nonequivalent charge and spin distributions as sketched in Figure 5. The imbalance of the spin density causes a rotation of the g-tensor by an angle of $\phi$. Our findings are in accord with the suggestion by Riera and Poilblanc [52] that charge order in TMTTF salts is a cooperative effect between the Coulomb interaction and the electronic coupling of the TMTTF stacks to the anions The charge disproportionation also causes an imbalance in the Coulomb attraction on the anions, which become slightly distorted in $b c$ plane in accord with thermal expansion results by de Souza et al. [53].

The occurrence of spontaneous dipole moments [26] and second harmonic generation [55] evidences the loss of inversion symmetry (with one center located at the anions, for instance) for $T<T_{\mathrm{CO}}$. Our vibrational spectra (Figure 1) show the presence of charge-rich and charge-poor molecules, which, however, can be arranged on the dimerized stacks in two possible ways: +-+-+- or -+-+-+ . This immediately implies that the rotation of the g-tensor can occur in both directions $\pm \phi$. Thus at $T<T_{\mathrm{CO}}$ domains are formed with inequivalent magnetic sites on neighboring stacks at the boundaries, as depicted in Figure 5. Without interaction two distinct ESR signals are expected below $T_{\mathrm{CO}}$, but the strong coupling leads to a significant broadening, that might get resolved by going to even higher frequencies.

Structural investigations could not identify any superstructure or change in symmetry, however, a strong increase in mosaicity at $T_{\mathrm{CO}}$, as shown in Figure 7 [54]. We conclude that the ordering occurs on a local scale with nanometersized CO domains. Similar, but larger ferroelectric domains of electronic origin have been directly observed in the twodimensional organic compound $\alpha$-(BEDT-TTF $)_{2} \mathrm{I}_{3}$ below the CO transition [56].

As the averaged g-tensor does not abruptly change at $T_{\mathrm{CO}}$, the charge ordering can be pictured as follows: For $T>$ $T_{\mathrm{CO}}$ random charge disproportionation exists on the TMTTF stacks giving rise to an averaged $g$-factor as observed, but without any anisotropic Zeeman effect, which is suppressed due to narrowing by large intrachain exchange. Below $T_{\mathrm{CO}}$, the charge imbalance becomes cooperative within each stack; there is some weak interaction of adjacent chains. At the boundary of two domains defined by the direction of charge and spin distortion, translational symmetry is lost. This gives rise to two inequivalent chains that yield the same averaged g-tensor along the $\tilde{a}$-Bindestrich and $\tilde{b}$-Bindestrich axes as above $T_{\mathrm{CO}}$. In the diagonal directions, however, the anisotropic Zeeman effect now becomes visible in our ESR data because the signal is narrowed by the much weaker interchain exchange.

\section{Conclusions}

In summary, the charge order observed in the linear-chain compounds (TMTTF) ${ }_{2} X$ by the splitting of the molecular vibrations opens a gap in the charge excitations. It also affects the magnetic behavior via mutual interaction with the anions. For $T<T_{\mathrm{CO}}$ the inversion symmetry is broken; charge disproportionation leads to a different electronic distortion on the organic molecules that eventually rotates the g-tensors, with two possible directions $\pm \phi$. From our multifrequency ESR measurements we conclude that in the charge-ordered regime two inequivalent magnetic TMTTF chains coexist, which produce a doubling in the angular periodicity of the linewidth as well as the characteristic quadratic frequency dependence. Thus, our experiments show that charge order not only causes ferroelectricity, but can also break the symmetry of the magnetic degree of freedom in quantum spin chains.

\section{Appendices}

\section{A. Structure}

The Fabre salts (TMTTF) ${ }_{2} X$ are charge transfer salts consisting of stacks of the planar organic molecules TMTTF (which stands for tetramethyltetrathiafulvalene) along the $a$-axis that are separated in $c$ direction by monovalent anions $X$. The overlap of the $\pi$ orbitals in a direction makes them model systems of one-dimensional metals. In $b$-direction the distance of the stacks is comparable to the van der Waals radii. Basically, no coupling between the molecular stacks exists in the $c$ direction. In the case of the Bechgaard salts the TMTSF molecule contains selenium instead of sulphur with more extended orbitals leading to better metallic conduction and even superconductivity [32, 57-59].

All compounds of the TMTTF family are isostructural (triclinic $P \overline{1}$ space group, $C_{i}$ symmetry with $Z=1$ ) with 

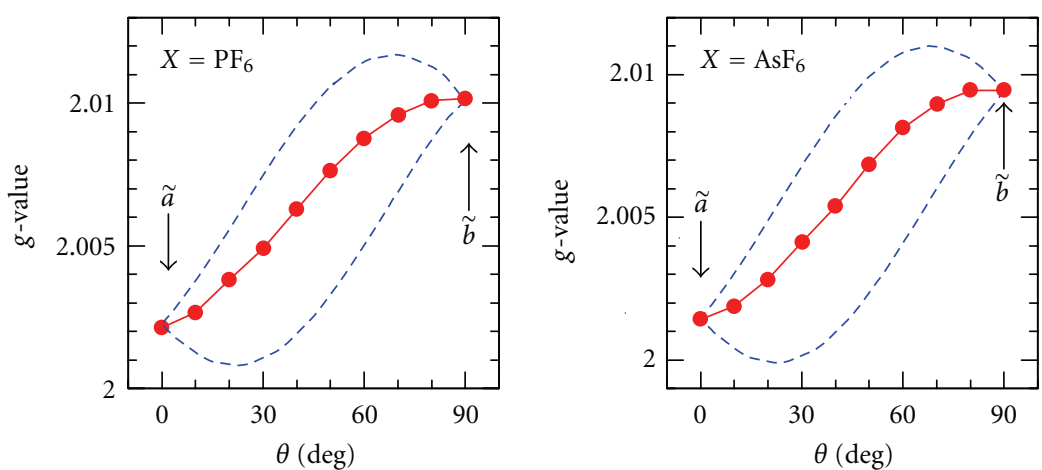

(a)

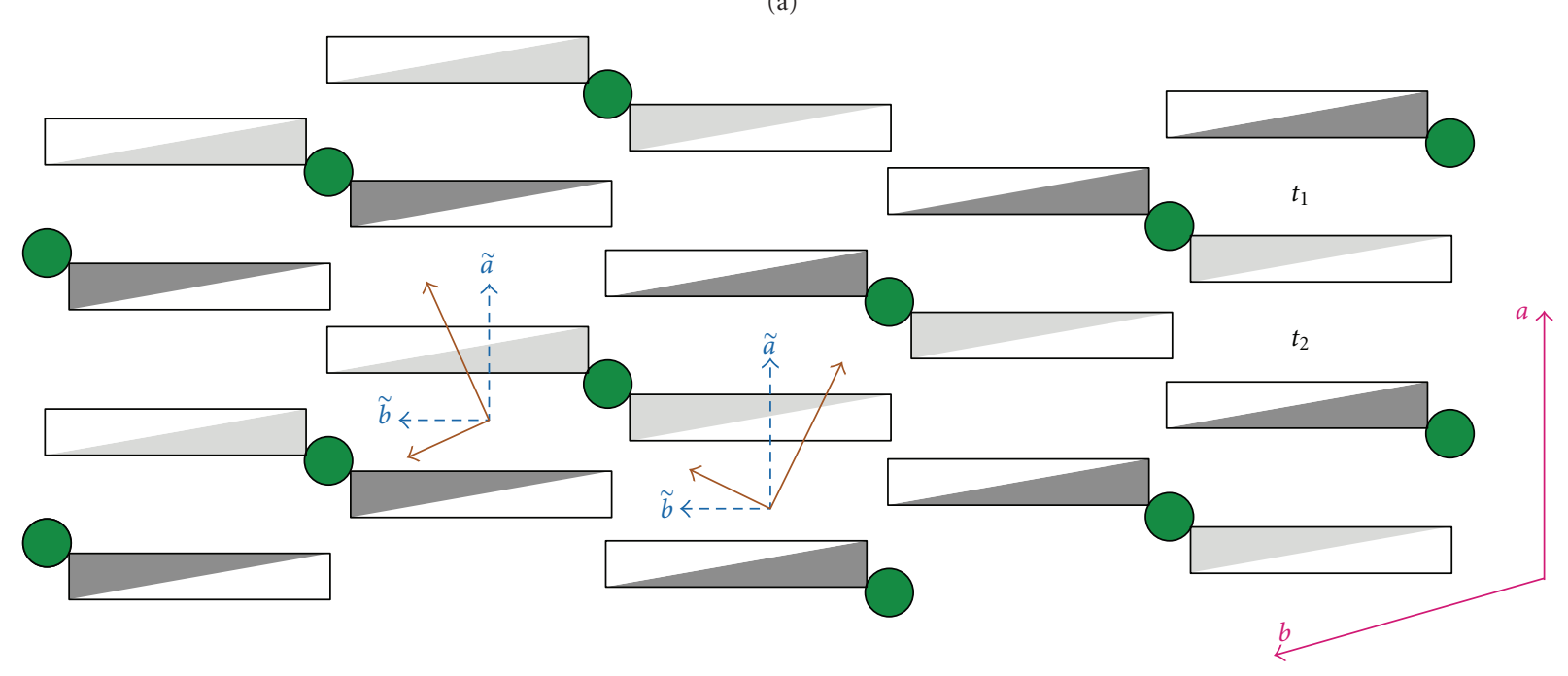

(b)

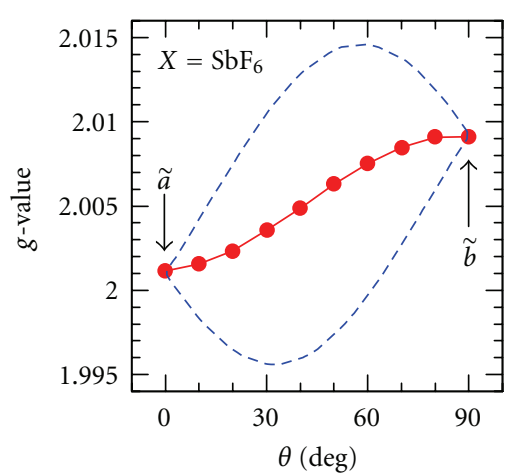

Figure 5: Angular dependence of the $g$-value of $(\mathrm{TMTTF})_{2} \mathrm{PF}_{6},(\mathrm{TMTTF})_{2} \mathrm{AsF}_{6}$, and $(\mathrm{TMTTF})_{2} \mathrm{SbF}_{6}$ evaluated at $T=30,50$, and $60 \mathrm{~K}$, respectively, when a static magnetic field $B_{0}$ is rotated in the $\tilde{a} \tilde{b}$ plane. The dashed lines indicate the calculated variation (equation $\mathrm{C} 1$ in the appendix) of the $g$-values of the two magnetically inequivalent sites. A view along the molecular axis $\tilde{c}$ is sketched in the lower panel. Below $T_{\mathrm{CO}}$ the charge distribution on the dimer becomes imbalanced (indicated by the different grey shades). The distinct coupling to the anions (green circles) modifies the electronic wavefunction causing the g-tensor to rotate in opposite directions, as depicted by the brown arrows. The transfer integrals $t_{1}$ and $t_{2}$ differ by about $40 \%$ at room temperature.

the TMTTF stacked normal to the molecular plane in $a$ direction, slightly displaced in $c$ direction to optimize the orbital overlap of the selenium atoms along $a$. In spite of the $A_{2} B$ stoichiometry and the corresponding dimerization, the separation of the TMTTF molecules along the stacks only differs by approximately $3 \%$ at room temperature and even less at low temperature $[28,60]$, implying almost equal overlap integrals along the $a$-axis. Due to the triclinic symmetry, $b^{\prime}$ commonly denotes the projection of the $b$ axis perpendicular to $a$, and $c^{*}$ is normal to the $a b$ plane. Here we introduce a Cartesian coordinate system linked to the TMTTF molecule, with $\tilde{a}$ points normal to the molecular plane, $\tilde{c}$ is the extended molecular axis, and $\tilde{b}$ is perpendicular to both (cf. inset of Figure 2). In a good approximation $\tilde{a}$ is parallel to the stacking axis $a$, and $\tilde{c}$ is almost $c+b$, as depicted in Figure 6 .

X-ray investigations of (TMTTF) ${ }_{2} \mathrm{SbF}_{6}$ single crystals performed at different temperatures down to $T=80 \mathrm{~K}$ rule out a structural transition at the charge-order temperature [54], confirming previous attempts by Coulon and collaborators $[27,60,61]$. Structural data do not unambiguously reveal the loss of inversion symmetry. Their analysis, however, yields a marginal better fit with no symmetry restrictions; but this also holds (to a minor degree) at room temperature. Charge order does not constitute a superstructure; nevertheless, the spots broaden significantly upon cooling with a distinct change at $T_{\mathrm{CO}}$. This is a measure of the mosaicity of the sample: in Figure 7 the temperature dependence of the mosaicity is plotted normalized to the room-temperature value. We interpret this as the development of domains with the $\mathrm{SbF}_{6}^{-}$anions slightly distorted in one or the other direction. The distortion, but also the domain size changes with temperature leading to a maximum around $T_{\mathrm{CO}}$. Typical for an effect of disorder, the phenomenon depends on cooling rate and slightly vary from crystal to crystal. 


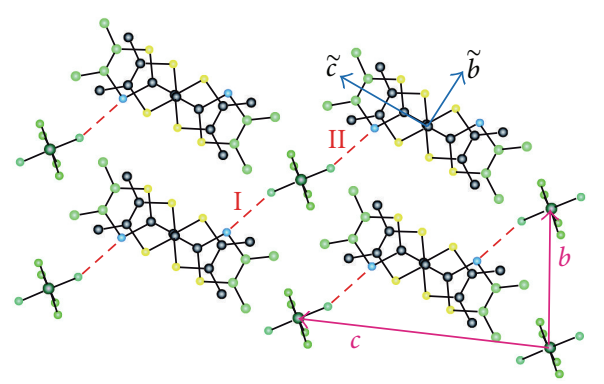

(a)

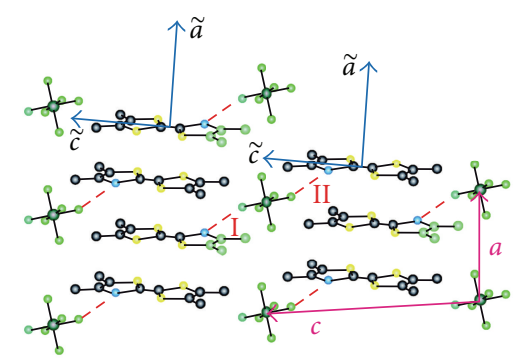

(b)

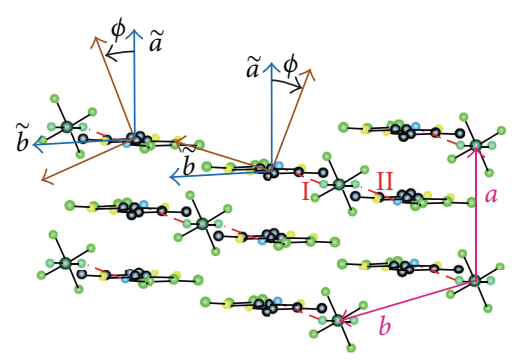

(c)

Figure 6: Crystal structure of (TMTTF) ${ }_{2} \mathrm{SbF}_{6}$, as an example, viewed (a) along the stacking direction $a$, (b) along the $b$-axis and (c) along the $c$ direction. The strongest coupling between the octahedral anions and the sulfur atoms is indicated by the red dashed lines I and II which are related by inversion symmetry for $T>T_{\mathrm{CO}}$. In the charge-ordered state, not only the TMTTF molecules develop a charge disproportionation with alternating charge-rich and charge-poor molecules along the stacks, but also the octahedral anions are slightly distorted. The inversion symmetry is lifted and contact I becomes stronger than II, for instance. Two kinds of domains are formed separated by domain walls preferentially in the $a b$ or $a c$ planes. Also shown is the configuration between the TMTTF molecular structure and the principal axes of the g-tensor. Note there exists only one electron per dimer. At elevated temperatures the g-tensor (blue arrows indicate the principal magnetic directions $\tilde{a}, \tilde{b}$, and $\widetilde{c}$ ) is determined by the molecular structure. The $\tilde{a}$-axis of the g-tensor is normal to the molecular plane and along the stacking direction, while the $\tilde{c}$-axis points along to the longest molecule extension. For $T<T_{\mathrm{CO}}$ the principal axes of the g-tensor rotate around the $\tilde{c}$-axis by an angle $\phi$ in opposite directions, resulting in the brown arrows.

\section{B. Experimental Details}

Single crystals of (TMTTF) ${ }_{2} X$ with $X=\mathrm{PF}_{6}, \mathrm{AsF}_{6}$ and $\mathrm{SbF}_{6}$ were grown by electrochemical methods in an H-type glass cell at room temperature [62]. A constant voltage of $1.5 \mathrm{~V}$ was applied between platinum electrodes with an area of approximately $1 \mathrm{~cm}^{2}$. The current through the solution was between 1 and $2 \mu \mathrm{A}$. After several weeks we were able to harvest needle-shaped single crystals of several millimeters in length and less than a millimeter in width.

The electrical transport was characterized by dc resistivity measured as a function of temperature in all three directions [28]. We evaporated small gold contacts onto the natural crystal surface and attached thin gold wires by carbon paste. Along the long $a$-axis of the crystals and also for the $b$ direction four-point measurements could be performed, while for the $c$ direction two contacts were applied on opposite sides of the crystal. The samples were attached to a sapphire plate in order to ensure good thermal contact and slowly cooled down to helium temperatures.

Measurements of the magnetic susceptibility were performed using a Quantum Design SQUID (superconducting quantum interference device) magnetometer between 1.8 and $380 \mathrm{~K}$. A large number of single crystals were glued parallel to each other inside a plastic straw using vacuum grease, and the magnetic field was applied parallel to the $a$ axis. The background signal of the sample holder and the vacuum grease was measured separately and subtracted in order to obtain the intrinsic magnetization of the sample. To estimate the spin susceptibility, we subtracted a temperature independent diamagnetic contribution of the core electrons $\chi_{\text {dia }}=-5.3 \times 10^{-9} \mathrm{~m}^{3} /$ mole from the original data of all specimen under consideration.

The infrared reflectivity spectra polarized along the $c$ direction were recorded by utilizing a Bruker IFS 66v/s and a Bruker Vertex 80 Fourier transform spectrometer extended with a IR microscope HYPERION which is purged with nitrogen to suppress any disturbing influence of the water absorption bands. The reflectivity spectra were measured in a frequency range from $500 \mathrm{~cm}^{-1}$ until $8000 \mathrm{~cm}^{-1}$ at temperatures from $10 \mathrm{~K}$ up to $290 \mathrm{~K}$. Here we focus on the perpendicular direction, that is $E \| c^{*}$. To receive the real optical conductivity, we performed a Kramers-Kronig analysis with a constant extrapolation for the low-frequency range and a $\omega^{-2}$ and $\omega^{-4}$ decrease for the high-frequency part. With a linear interpolation between the mode frequency in fully neutral $\left(1628 \mathrm{~cm}^{-1}\right)$ and fully ionized $\left(1548 \mathrm{~cm}^{-1}\right)$ TMTTF molecules [63], we can quantitatively determine the charge imbalance $\left[2 \delta=\Delta v /\left(80 \mathrm{~cm}^{-1} / \mathrm{e}\right)\right]$, and the molecular ionicity.

The electron spin resonance (ESR) spectra were carried out in a continuous wave X-band spectrometer Bruker ESP 300 at $9.5 \mathrm{GHz}$ and a W-band spectrometer Bruker Elexsys 680 at $95 \mathrm{GHz}$ at Stuttgart University, and a Qband spectrometer Bruker Elexsys 500 at $34 \mathrm{GHz}$ at Augsburg University. Complementary X-band measurements were performed at the Hochfeld-Magnetlabor Dresden. The temperature dependence of the ESR properties was measured down to $T=4 \mathrm{~K}$ by utilizing continuous-flow helium cryostats $[29,30]$. The typical size of the single crystals used in the X-band ESR measurements was $2 \times 0.5 \times 0.1 \mathrm{~mm}^{3}$, while very small samples of less than $0.4 \times 0.4 \times 0.05 \mathrm{~mm}^{3}$ were used in the Q- and W-band ESR measurements in order to avoid any sample-size effect that can lead to a broadening of the linewidth [41].

\section{Magnetic Characterization}

The large thermal expansion of the organic compounds has strong effects on the temperature dependence of the spin susceptibility. To compare the experimental results (usually 


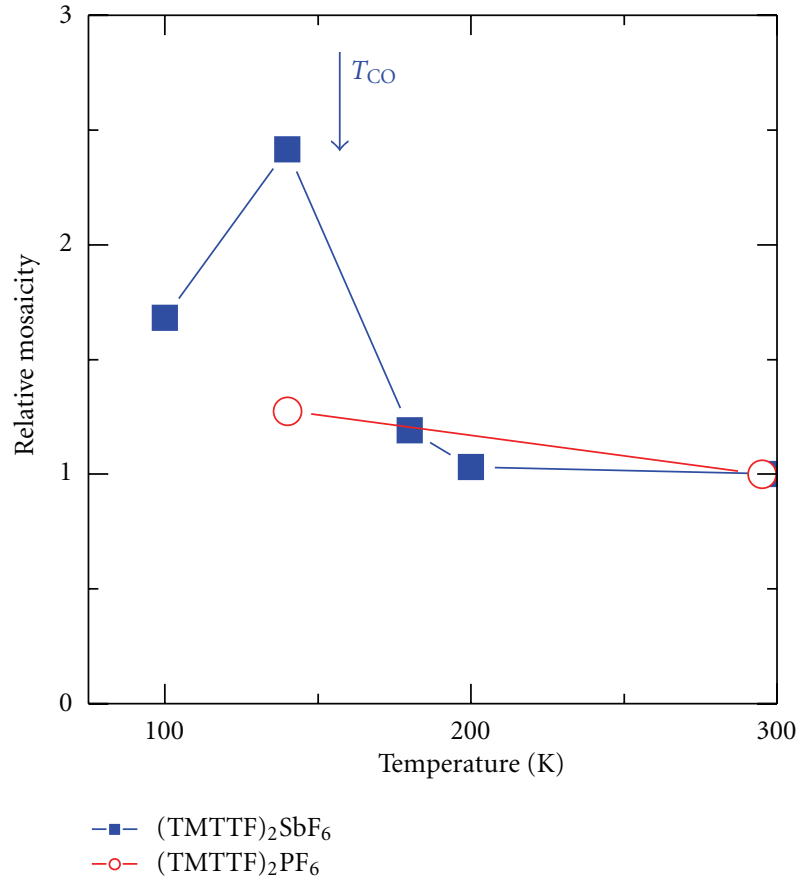

FIGURE 7: Temperature dependence of the normalized mosaicity of (TMTTF) ${ }_{2} \mathrm{SbF}_{6}$ and (TMTTF) ${ }_{2} \mathrm{PF}_{6}$ determined from the width of the X-ray spots (after [54]). When the temperature drops below $200 \mathrm{~K}$, the mosaicity of (TMTTF) ${ }_{2} \mathrm{SbF}_{6}$ increases dramatically approaching a maximum at the charge-order temperature $T_{\mathrm{CO}}=$ $157 \mathrm{~K}$. For comparison the behavior of (TMTTF) ${ }_{2} \mathrm{PF}_{6}$ is also presented where only a small increase is observed. Note, in the case of (TMTTF $)_{2} \mathrm{PF}_{6}$ the phase transition happens only at $T_{\mathrm{CO}}=67 \mathrm{~K}$.

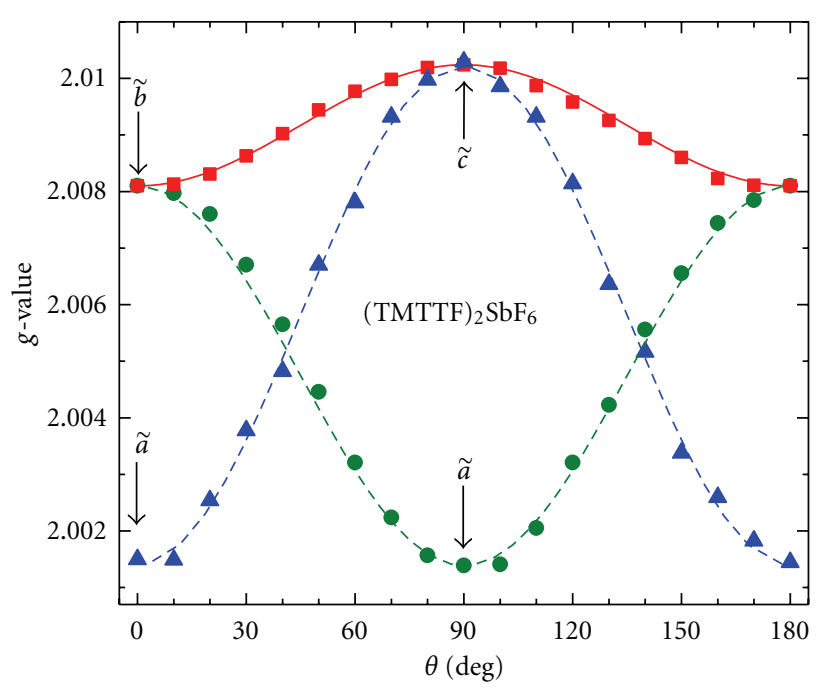

Figure 8: Angular dependence of the $g$-value of (TMTTF) ${ }_{2} \mathrm{SbF}_{6}$ at room temperature measured at X-band frequency when the static magnetic field $B_{0}$ is rotated within the $\tilde{a} \tilde{b}$ plane (green circles), the $\tilde{b} \tilde{c}$ plane (red squares), and the $\tilde{a} \tilde{c}$ plane (blue triangles). The leastsquare fits by $g(\theta)=\left(g_{\min }^{2} \cos ^{2} \theta+g_{\max }^{2} \sin ^{2} \theta\right)^{1 / 2}$ are shown by the corresponding lines. obtained at $p=$ const) with the theoretical predictions (in general calculated for $V=$ const), the spin susceptibility at constant pressure $\left(\chi_{s}\right)_{p}$ has to be transformed in the spin susceptibility at constant volume $\left(\chi_{s}\right)_{V}$. In the case of (TMTSF $)_{2} \mathrm{PF}_{6}$ the temperature dependence of $\left(\chi_{s}\right)_{V}$ was estimated by Wzietek et al. [64] from NMR and Xray measurements under pressure. We assumed that the substitution of sulfur for selenium and the exchange of the inorganic anions has no considerable influence on the thermal expansion, thus we took the ratio $\left(\chi_{s}\right)_{V} /\left(\chi_{s}\right)_{p}$ for different temperatures to rescale our susceptibility data of $(\mathrm{TMTTF})_{2} X$. At high temperatures the spin susceptibility at constant volume of (TMTTF) ${ }_{2} X$ resembles the well-known behavior of a spin-1/2 Heisenberg chain with antiferromagnetic coupling as depicted in Figure 1 by the dashed blue lines. The thermodynamic and magnetic properties of such a system were studied by Bonner and Fisher [65]. The advanced model of Eggert, Affleck, and Takahashi [8] using the Bethe ansatz differs appreciably only at low temperatures $(T<0.2 \mathrm{~J})$. For $T>100 \mathrm{~K}$ the ESR intensity at constant volume can be modeled using $J \approx 420,410$, and $400 \mathrm{~K}$ for $(\mathrm{TMTTF})_{2} \mathrm{PF}_{6}$, (TMTTF) ${ }_{2} \mathrm{AsF}_{6}$, and (TMTTF $)_{2} \mathrm{SbF}_{6}$, respectively.

The temperature dependence of the ESR results along the three crystal axes of (TMTTF) ${ }_{2} \mathrm{PF}_{6}$, (TMTTF) ${ }_{2} \mathrm{AsF}_{6}$, and $(\mathrm{TMTTF})_{2} \mathrm{SbF}_{6}$ are shown in Figure 9. The $g$-shift $\Delta g(T)=g(T)-2.002319$ for all three crystal axes is very small. At elevated temperatures, the $g$-values show no significant temperature dependence but a distinct anisotropy with a negative value of $\Delta g$ along the chain direction $\widetilde{a}$ and positive values perpendicular to the chain direction, as seen in Figure 8. This behavior is found in all TMTTF and TMTSF salts as presented in $[29,30]$. In all cases the small anisotropy of $\Delta g$ between the $\widetilde{b}$ - and the $\tilde{c}$-axes increases with temperature. The $g$-value is largest for $B_{0} \| \tilde{c}$ and closest to the free-electron value parallel to the stacks. As the temperature is lowered, the linewidth $\Delta H$ decreases almost linearly with $T$ in all directions. In the $\tilde{c}$ direction $\Delta H(T)$ is slightly larger; the most narrow lines are always observed along the chains.

At low temperatures (TMTTF) ${ }_{2} \mathrm{PF}_{6}$ and (TMTTF) ${ }_{2} \mathrm{AsF}_{6}$ undergo spin-Peierls transitions at $T_{\mathrm{SP}}=19$ and $13 \mathrm{~K}$, respectively. The formation of states with singlet-paired holes due to the tetramerization causes a decreasing susceptibility with an exponential drop of the intensity down to lowest temperatures, but it does not completely vanish (Figure 1). This phase transition also leads to a broadening of the ESR linewidth and a small change in the $g$-values [29]. $(\mathrm{TMTTF})_{2} \mathrm{SbF}_{6}$, on the other hand, orders antiferromagnetically at $T_{N}=8 \mathrm{~K}$, leading to a decrease in $\chi_{s}(T)$ with decreasing temperature before it eventually turns up again. This phase transition is accompanied by a decrease in the $g$-shift for the $\tilde{b}$ and $\tilde{c}$ axes; along the $\tilde{a}$-axis the $g$-factor becomes larger below $T \approx 15 \mathrm{~K}$. The low-temperature ordered states are out of the scope of the present paper and discussed elsewhere $[29,66]$.

From the fact that the maximum of $\Delta H$ occurs at the diagonal direction $\theta=45^{\circ}$ of the $a b$ plane and that the $g$ values of neighboring molecules differ by $\Delta g$, we conclude 

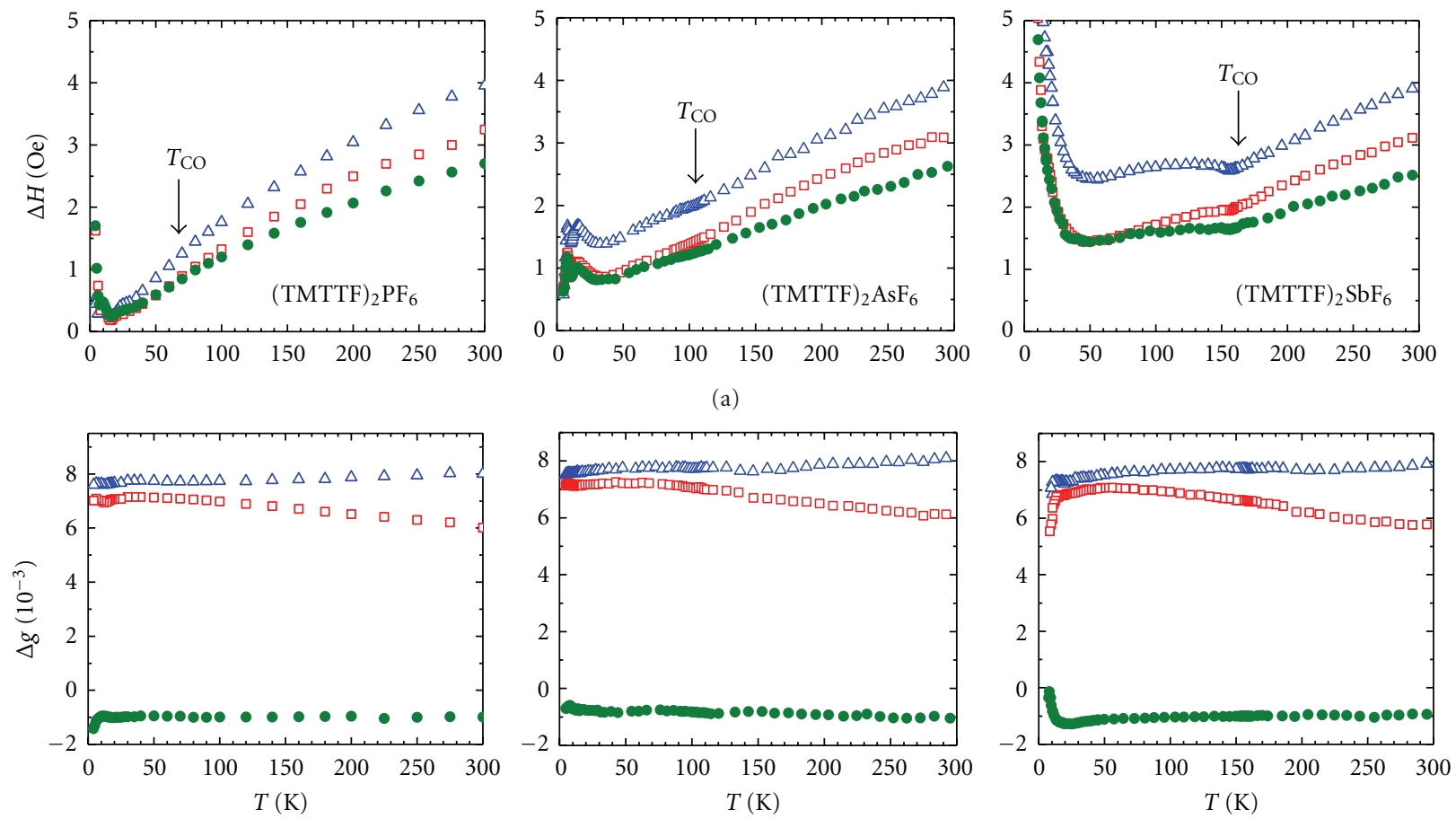

$$
\begin{aligned}
& \Delta \tilde{c} \text {-axis } \\
& \square \tilde{b} \text {-axis } \\
& \text { - } \tilde{a} \text {-axis }
\end{aligned}
$$$$
\Delta \tilde{c} \text {-axis }
$$$$
\text { ㄷ } \tilde{b} \text {-axis }
$$$$
\Delta \tilde{c} \text {-axis }
$$$$
\text { - } \tilde{b} \text {-axis }
$$

- $\tilde{a}$-axis

- $\tilde{a}$-axis

(b)

Figure 9: Temperature dependence of (a) the ESR linewidth $\Delta H$, and (b) the $g$-shift $\Delta g(T)=g(T)-2.002319$ for $\left(\mathrm{TMTTF}_{2} \mathrm{PF}_{6}(\mathrm{left}\right.$ panel), (TMTTF) ${ }_{2} \mathrm{AsF}_{6}$ (middle panel) and (TMTTF) ${ }_{2} \mathrm{SbF}_{6}$ (right panel) measured by X-band spectroscopy along the three directions $\tilde{a}$ (green dots), $\tilde{b}$ (open red squares), and $\tilde{c}$ (blue triangles).
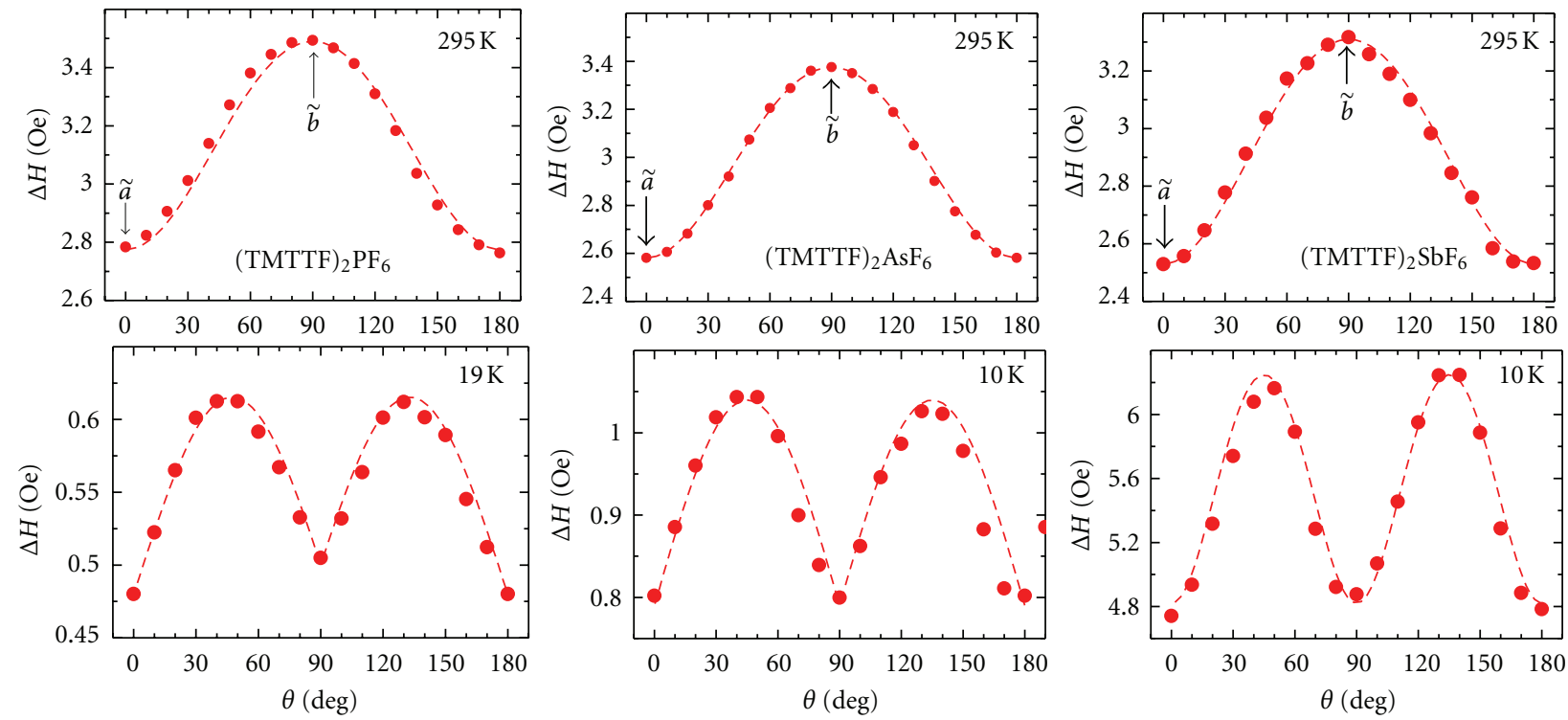

FIgURe 10: Angular dependence of the X-band ESR linewidth within the $\tilde{a} \tilde{b}$ plane of $\left(\mathrm{TMTTF}_{2} \mathrm{PF}_{6}\left(\right.\right.$ left panel), $(\mathrm{TMTTF})_{2} \mathrm{AsF}_{6}($ middle panel), and (TMTTF) ${ }_{2} \mathrm{SbF}_{6}$ (right panel) measured at (a) room temperature and (b) low temperature, as indicated. The solid lines are obtained from fits of the data by (1) and (2). Note the different vertical scales, indicating the observed effect is about ten times as big in (TMTTF) $)_{2} \mathrm{SbF}_{6}$ compared to (TMTTF) ${ }_{2} \mathrm{PF}_{6}$. 
that the g-tensors of the spins corresponding to the two different magnetic sites do not coincide with the principle magnetic axes. They are rotated around the $\tilde{c}$-axis by $\phi$ in opposite directions as depicted in Figure 6. As shown in Figure 5, the two different sites contribute to the observed $g$-value according to

$$
\begin{aligned}
& g_{1}=\left(g_{\max }^{2} \cos ^{2}\{\theta-\phi\}+g_{\min }^{2} \sin ^{2}\{\theta-\phi\}\right)^{1 / 2}, \\
& g_{2}=\left(g_{\max }^{2} \cos ^{2}\{\theta+\phi\}+g_{\min }^{2} \sin ^{2}\{\theta+\phi\}\right)^{1 / 2},
\end{aligned}
$$

where $g_{1}$ and $g_{2}$ correspond to the g-tensor of type 1 and type 2 spins, respectively. The principal axes of the two kinds of spins span the angle $\phi . g_{\max }$ and $g_{\min }$ are the maximum and the minimum of the $g$-values for each kind of spins at the angle $\phi$, respectively. For symmetry reasons the $g$ values have to be identical $g_{1}=g_{2}$ at $\theta=0^{\circ}$ and $\theta=$ $90^{\circ}$, while at $\theta=45^{\circ}$ the difference of the $g$-values is obtained from the anisotropic Zeeman effect. We obtain for $(\mathrm{TMTTF})_{2} \mathrm{PF}_{6} g_{\max }=2.0117, g_{\min }=2.0008$, and $\phi=22^{\circ}$; for $(\mathrm{TMTTF})_{2} \mathrm{AsF}_{6} g_{\max }=2.0071, g_{\min }=1.9999$, and $\phi=$ $22^{\circ}$; and $g_{\max }=2.0143, g_{\min }=1.9956$, and $\phi=32^{\circ}$, for the example of (TMTTF) $)_{2} \mathrm{SbF}_{6}$.

In Figure 10 the linewidth of $(\mathrm{TMTTF})_{2} \mathrm{PF}_{6}$. $(\mathrm{TMTTF})_{2} \mathrm{AsF}_{6}$, and $(\mathrm{TMTTF})_{2} \mathrm{SbF}_{6}$ is plotted as a function of angle when the magnetic field is rotated around the $\tilde{c}$-axis. While at room temperature (upper frames) the angular dependence follows (1), $\Delta H$ strongly increases in the diagonal direction $\left(\phi= \pm 45^{\circ}\right)$ as $T<T_{\mathrm{CO}}$ (lower frames). The effect becomes stronger when going from (TMTTF) ${ }_{2} \mathrm{PF}_{6}$ to $(\mathrm{TMTTF})_{2} \mathrm{AsF}_{6}$ and to (TMTTF $)_{2} \mathrm{SbF}_{6}$, corresponding to the increase in charge disproportionation and transition temperature $T_{\mathrm{CO}}$.

\section{Acknowledgments}

The authors thank S. Brown, A. Jánossy, J.-P. Pouget, and E. Rose for useful discussions. The crystals were grown by $\mathrm{G}$. Untereiner, H.J. Kümmerer, H.-A. Krug von Nidda, F. Lissner, and M. Ozerov assisted during some experiments. The work is supported by the Deutsche Forschungsgemeinschaft (DFG). T. Knoblauch acknowledges a fellowship of the CarlZeiss-Stiftung.

\section{References}

[1] M. Imada, A. Fujimori, and Y. Tokura, "Metal-insulator transitions," Reviews of Modern Physics, vol. 70, no. 4, pp. 1039-1263, 1998.

[2] H. Seo, C. Hotta, and H. Fukuyama, "Toward systematic understanding of diversity of electronic properties in lowdimensional molecular solids," Chemical Reviews, vol. 104, no. 11, pp. 5005-5036, 2004.

[3] J. M. Tranquada, B. J. Sternlieb, J. D. Axe, Y. Nakamura, and S. Uchida, "Evidence for stripe correlations of spins and holes in copper oxide superconductors," Nature, vol. 375, no. 6532, pp. 561-563, 1995.

[4] S. A. Kivelson, I. P. Bindloss, E. Fradkin et al., "How to detect fluctuating stripes in the high-temperature superconductors," Reviews of Modern Physics, vol. 75, no. 4, pp. 1201-1241, 2003.
[5] J. Merino and R. H. McKenzie, "Superconductivity mediated by charge fluctuations in layered molecular crystals," Physical Review Letters, vol. 87, no. 23, Article ID 237002, 4 pages, 2001.

[6] M. Dressel, N. Drichko, J. Schlueter, and J. Merino, "Proximity of the layered organic conductors $\alpha$-(BEDT$\mathrm{TTF})_{2} \mathrm{MHg}(\mathrm{SCN})_{4}\left(M=\mathrm{K}, \mathrm{NH}_{4}\right)$, to a charge-ordering transition," Physical Review Letters, vol. 90, no. 16, Article ID 167002, 4 pages, 2003.

[7] S. Kaiser, M. Dressel, Y. Sun et al., "Bandwidth tuning triggers interplay of charge order and superconductivity in twodimensional organic materials," Physical Review Letters, vol. 105, no. 20, Article ID 206402, 4 pages, 2010.

[8] S. Eggert, I. Affleck, and M. Takahashi, "Susceptibility of the spin 1/2 Heisenberg antiferromagnetic chain," Physical Review Letters, vol. 73, no. 2, pp. 332-335, 1994.

[9] N. Ikeda, H. Ohsumi, K. Ohwada et al., "Ferroelectricity from iron valence ordering in the charge-frustrated system LuFe2O4," Nature, vol. 436, no. 7054, pp. 1136-1138, 2005.

[10] D. Niermann, F. Waschkowski, J. De Groot, M. Angst, and J. Hemberger, "Dielectric properties of charge-ordered $\mathrm{LuFe}_{2} \mathrm{O}_{4}$ revisited: the apparent influence of contacts," Physical Review Letters, vol. 109, no. 1, Article ID 016405, 5 pages, 2012.

[11] M. Alexe, M. Ziese, D. Hesse et al., "Ferroelectric switching in multiferroic magnetite $\left(\mathrm{Fe}_{3} \mathrm{O}_{4}\right)$ thin films," Advanced Materials, vol. 21, no. 44, pp. 4452-4455, 2009.

[12] K. Yamauchi, T. Fukushima, and S. Picozzi, "Ferroelectricity in multiferroic magnetite $\mathrm{Fe}_{3} \mathrm{O}_{4}$ driven by noncentrosymmetric $\mathrm{Fe}^{2+} / \mathrm{Fe}^{3+}$ charge-ordering: first-principles study," Physical Review B, vol. 79, no. 21, Article ID 212404, 4 pages, 2009.

[13] K. Yamauchi and S. Picozzi, "Interplay between charge order, ferroelectricity, and ferroelasticity: tungsten bronze structures as a playground for multiferroicity," Physical Review Letters, vol. 105, no. 10, Article ID 107202, 4 pages, 2010.

[14] F. Schrettle, S. Krohns, P. Lunkenheimer, V. A. M. Brabers, and A. Loidl, "Relaxor ferroelectricity and the freezing of shortrange polar order in magnetite," Physical Review B, vol. 83, no. 19, Article ID 195109, 5 pages, 2011.

[15] A. Ruff, S. Krohns, F. Schrettle, V. Tsurkan, P. Lunkenheimer, and A. Loidl, "Absence of polar order in $\mathrm{LuFe}_{2} \mathrm{O}_{4}$," European Physical Journal B, vol. 85, no. 8, article 290, 2012.

[16] D. V. Efremov, J. Van Den Brink, and D. I. Khomskii, "Bondversus site-centred ordering and possible ferroelectricity in manganites," Nature Materials, vol. 3, no. 12, pp. 853-856, 2004.

[17] Y. J. Choi, H. T. Yi, S. Lee, Q. Huang, V. Kiryukhin, and S.-W. Cheong, "Ferroelectricity in an ising chain magnet," Physical Review Letters, vol. 100, no. 4, Article ID 047601, 4 pages, 2008.

[18] R. Ramesh and N. A. Spaldin, "Multiferroics: progress and prospects in thin films," Nature Materials, vol. 6, no. 1, pp. 2129, 2007.

[19] S. W. Cheong and M. Mostovoy, "Multiferroics: a magnetic twist for ferroelectricity," Nature Materials, vol. 6, no. 1, pp. 13-20, 2007.

[20] N. A. Hill, "Why are there so few magnetic ferroelectrics?" Journal of Physical Chemistry B, vol. 104, no. 29, pp. 66946709, 2000.

[21] D. I. Khomskii, "Magnetism and ferroelectricity: why do they so seldom coexist?" Bulletin of the American Physical Society, vol. 21, article 002, 2001.

[22] J. Van Den Brink and D. I. Khomskii, "Multiferroicity due to charge ordering," Journal of Physics Condensed Matter, vol. 20, no. 43, Article ID 434217, 2008. 
[23] T. Kimura, T. Goto, H. Shintani, K. Ishizaka, T. Arima, and Y. Tokura, "Magnetic control of ferroelectric polarization," Nature, vol. 426, no. 6962, pp. 55-58, 2003.

[24] H. Schmid, "Some symmetry aspects of ferroics and single phase multiferroics," Journal of Physics Condensed Matter, vol. 20, no. 43, Article ID 434201, 2008.

[25] D. I. Khomskii, “Trend: classifying multiferroics: mechanisms and effects," Physics, vol. 2, article 20, 2009.

[26] P. Monceau, F. Y. Nad, and S. Brazovskii, "Ferroelectric mottHubbard phase of organic (TMTTF)2X conductors," Physical Review Letters, vol. 86, no. 18, pp. 4080-4083, 2001.

[27] C. Coulon, S. S. P. Parkin, and R. Laversanne, "Structureless transition and strong localization effects in bistetramethyltetrathiafulvalenium salts $\left[(\mathrm{TMTTF})_{2} X\right]$," Physical Review B, vol. 31, no. 6, pp. 3583-3587, 1985.

[28] B. Köhler, E. Rose, M. Dumm, G. Untereiner, and M. Dressel, "Comprehensive transport study of anisotropy and ordering phenomena in quasi-one-dimensional (TMTTF) ${ }_{2} X$ salts $\left(X=\mathrm{PF}_{6}, \mathrm{AsF}_{6}, \mathrm{SbF}_{6}, \mathrm{BF}_{4}, \mathrm{ClO}_{4}, \mathrm{ReO}_{4}\right), "$ Physical Review $B$, vol. 84, no. 3, Article ID 035124, 13 pages, 2011.

[29] M. Dumm, A. Loidl, B. W. Fravel, K. P. Starkey, L. K. Montgomery, and M. Dressel, "Electron spin resonance studies on the organic linear-chain compounds (TMTCF $)_{2} X(C=\mathrm{S}$,Se; $\left.X=\mathrm{PF}_{6}, \mathrm{AsF}_{6}, \mathrm{ClO}_{4}, \mathrm{Br}\right)$," Physical Review B, vol. 61, no. 1, pp. 511-521, 2000.

[30] B. Salameh, S. Yasin, M. Dumm, G. Untereiner, L. Montgomery, and M. Dressel, "Spin dynamics of the organic linear chain compounds (TMTTF $)_{2} X\left(X=\mathrm{SbF}_{6}, \mathrm{AsF}_{6}, \mathrm{BF}_{4}, \mathrm{ReO}_{4}\right.$, and SCN)," Physical Review B, vol. 83, no. 20, Article ID 205126, 8 pages, 2011.

[31] J. P. Pouget and S. Ravy, "Structural aspects of the Bechgaard salts and related compounds," Journal de Physique I, vol. 6, no. 12, pp. 1501-1525, 1996.

[32] D. Jérome and H. J. Schulz, "Organic conductors and superconductors," Advances in Physics, vol. 31, no. 4, pp. 299-490, 1982.

[33] L. Ducasse, M. Abderrabba, J. Hoarau, M. Pesquer, B. Gallois, and J. Gaultier, "Temperature dependence of the transfer integrals in the (TMTSF) ${ }_{2} X$ and (TMTTF) ${ }_{2} X$ families," Journal of Physics C, vol. 19, article 3805, 1986.

[34] M. Dressel, "Spin-charge separation in quasi one-dimensional organic conductors," Naturwissenschaften, vol. 90, no. 8, pp. 337-344, 2003.

[35] M. Dressel, "Ordering phenomena in quasi-one-dimensional organic conductors," Naturwissenschaften, vol. 94, no. 7, pp. 527-541, 2007.

[36] D. S. Chow, F. Zamborszky, B. Alavi et al., "Charge ordering in the TMTTF family of molecular conductors," Physical Review Letters, vol. 85, no. 8, pp. 1698-1701, 2000.

[37] F. Zamborszky, W. Yu, W. Raas et al., "Competition and coexistence of bond and charge orders in (TMTTF $)_{2} \mathrm{AsF}_{6}$," Physical Review B, vol. 66, no. 8, Article ID 081103, 4 pages, 2002.

[38] M. Dumm, B. Salameh, M. Abaker, L. K. Montgomery, and M. Dressel, "Magnetic and optical studies of spin and charge ordering in (TMTTF $)_{2} \mathrm{AsF}_{6}$," Journal de Physique IV, vol. 114, pp. 57-60, 2004.

[39] M. Dumm, M. Abaker, and M. Dressel, "Mid-infrared response of charge-ordered quasi-10 organic conductors (TMTTF $)_{2} X$," Journal de Physique IV, vol. 131, pp. 55-58, 2005.
[40] T. Knoblauch and M. Dressel, "Charge disproportionation in $(\mathrm{TMTTF})_{2} X\left(X=\mathrm{PF}_{6}, \mathrm{AsF}_{6}\right.$ and $\left.\mathrm{SbF}_{6}\right)$ investigated by infrared spectroscopy," Physica Status Solidi (C), vol. 9, no. 5, pp. 1158-1160, 2012.

[41] S. Yasin, B. Salameh, E. Rose et al., "Broken magnetic symmetry due to charge-order ferroelectricity discovered in (TMTTF $)_{2} X$ salts by multifrequency ESR," Physical Review B, vol. 85, no. 14, Article ID 144428, 14 pages, 2012.

[42] Y. Tanaka and M. Ogata, "Effects of charge ordering on the spin degrees of freedom is studied by using one-dimensional extended Hubbard model," Journal of the Physical Society of Japan, vol. 74, pp. 3283-3287, 2006.

[43] C. Coulon and R. Clerac, "Electron spin resonance: a major probe for molecular conductors," Chemical Reviews, vol. 104, no. 11, pp. 5655-5688, 2004.

[44] K. Furukawa, T. Hara, and T. Nakamura J, "Anomalous temperature dependence of g-tensor in organic conductor, (TMTTF $)_{2} X\left(X=\mathrm{Br}, \mathrm{PF}_{6}\right.$, and $\left.\mathrm{SbF}_{6}\right)$," Journal of the Physical Society of Japan, vol. 78, Article ID 104713, 6 pages, 2009.

[45] T. Nakamura, "Possible charge ordering patterns of the paramagnetic insulating states in (TMTTF) ${ }_{2} X$," Journal of the Physical Society of Japan, vol. 72, no. 2, pp. 213-216, 2003.

[46] A. Bencini and D. Gatteschi, Electron Paramagnetic Resonance of Exchange Coupled Systems, Springer, Berlin, Germany, 1990.

[47] M. Oshikawa and I. Affleck, "Low-temperature electron spin resonance theory for half-integer spin antiferromagnetic chains," Physical Review Letters, vol. 82, no. 25, pp. 5136-5139, 1999.

[48] M. Oshikawa and I. Affleck, "Electron spin resonance in $S=$ 1/2 antiferromagnetic chains," Physical Review B, vol. 65, no. 13, Article ID 134410, 8 pages, 2002.

[49] M. Herak, A. Zorko, D. Arčon et al., "Symmetric and antisymmetric exchange anisotropies in quasi-one-dimensional $\mathrm{CuSe}_{2} \mathrm{O}_{5}$ as revealed by ESR," Physical Review B, vol. 84, no. 18, Article ID 184436, 8 pages, 2011.

[50] B. Pilawa, "Anisotropy of the electron spin-resonance linewidth of $\mathrm{CuGeO}_{3}$," Journal of Physics, vol. 9, no. 18, p. 3779, 1997.

[51] C. Yasuda, S. Todo, K. Hukushima et al., "Néel temperature of quasi-low-dimensional Heisenberg antiferromagnets," Physical Review Letters, no. 94, Article ID 217201, 4 pages, 2005.

[52] J. Riera and D. Poilblanc, "Influence of the anion potential on the charge ordering in quasi-one-dimensional charge-transfer salts," Physical Review B, vol. 63, Article ID 241102, 4 pages, 2001.

[53] M. de Souza, P. Foury-Leylekian, A. Moradpour, J.-P. Pouget, and M. Lang, "Evidence for lattice effects at the chargeordering transition in (TMTTF) $)_{2} X$," Physical Review Letters, vol. 101, Article ID 216403, 4 pages, 2008.

[54] E. Rose, F. Lissner, T. Schleid, and M. Dressel, to be published.

[55] K. Yamamoto, S. Iwai, N. Nishi, and K. Yakushi, unpublished.

[56] K. Yamamoto, A. Kowalska, and K. Yakushi, "Current orientation and contact distance dependence of rapid voltage oscillations in the organic conductor $\beta^{\prime \prime}$-[bis(ethylenedithio) tetrathiafulvalene $]_{3}\left(\mathrm{HSO}_{4}\right)_{2}$," Applied Physics Letters, vol. 96, Article ID 122901, 6 pages, 2010.

[57] D. Jérome, "Organic superconductors: from (TMTSF) ${ }_{2} \mathrm{PF}_{6}$ to fullerenes," in Organic Conductors, J.-P. Farges, Ed., p. 405, Marcel Dekker, New York, NY, USA, 1994.

[58] T. Ishiguro, K. Yamaji, and G. Saito, Organic Superconductors, Springer, Berlin, Germnay, 2nd edition, 1998.

[59] S. A. Brazovskii, "Ferroelectricity and charge ordering in quasi-1D organic conductors," in The Physics of Organic 
Superconductors and Conductors, A. G. Lebed, Ed., p. 313, Springer, Berlin, Germany, 2008.

[60] R. Laversanne, C. Coulon, B. Gallois, J. P. Pouget, and R. Moret, "Structural and electrical properties of (TMTTF) ${ }_{2} \mathrm{MF}_{6}$ salts $(\mathrm{M}=\mathrm{P}, \mathrm{As}, \mathrm{Sb})$. Role of the anions," Journal de Physique Lettres, vol. 45, no. 8, pp. L393-L399, 1984.

[61] T. Granier, B. Gallois, A. Fritsch, L. Ducasse, and C. Coulon, "135 K crystallographic and electronic structure of (TMTTF $)_{2} \mathrm{SbF}_{6}$," in Lower-Dimensional Systems and Molecular Electronics, R. M. Metzger, P. Day, and G. C. Papavassiliou, Eds., vol. 248 of NATO Advanced Study Institute, Series B, p. 163, Plenum Press, New York, NY, USA, 1990.

[62] L. K. Montgomery, "Chemical synthesis and crystal growth techniques," in Organic Conductors, J. P. Farges, Ed., p. 138, Marcel Dekker, New York, NY, USA, 1994.

[63] M. Meneghetti, R. Bozio, I. Zanon, C. Pecile, C. Ricotta, and M. Zanetti, "Vibrational behavior of molecular constituents of organic superconductors: TMTSF, its radical cation and the sulphur analogs TMTTF and TMTTF+," The Journal of Chemical Physics, vol. 80, no. 12, pp. 6210-6224, 1983.

[64] P. Wzietek, C. Bourbonnais, F. Creuzet, D. Jérome, K. Bechgaard, and P. Batail, "Nuclear-relaxation and electronic correlations in quasi-one-dimensional organic conductors: 2. Experiments," Journal de Physique I, vol. 3, p. 171, 1993.

[65] J. C. Bonner and M. E. Fisher, "Linear magnetic chains with anisotropic coupling," Physical Review, vol. 135, no. 3, pp. A640-A658, 1964.

[66] M. Dumm, A. Loidl, B. Alavi, K. P. Starkey, M. Montgomery, and M. Dressel, "Comprehensive ESR study of the antiferromagnetic ground states in the one-dimensional spin systems (TMTSF $)_{2} \mathrm{PF}_{6},(\mathrm{TMTSF})_{2} \mathrm{AsF}_{6}$, and (TMTTF) ${ }_{2} \mathrm{Br}$," Physical Review B, vol. 62, no. 10, pp. 6512-6520, 2000. 

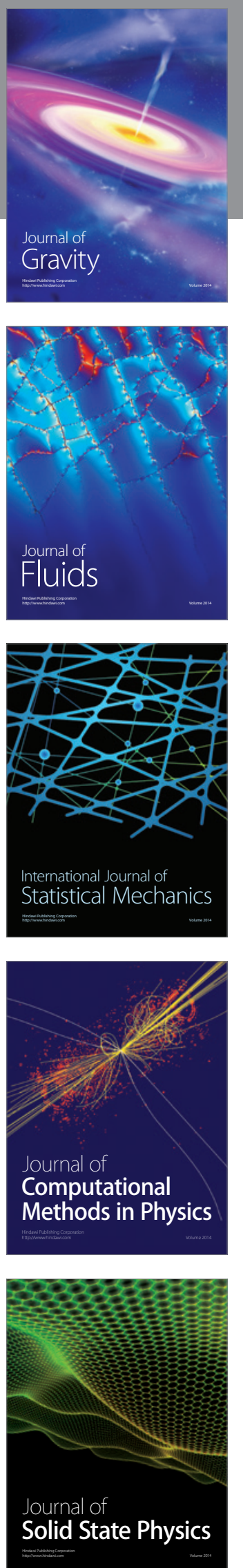

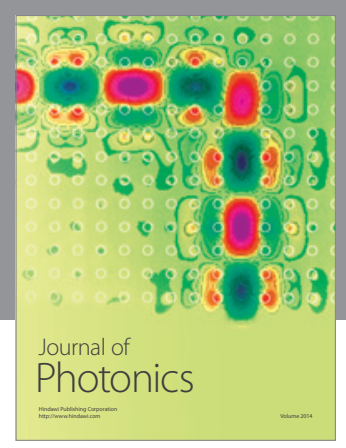

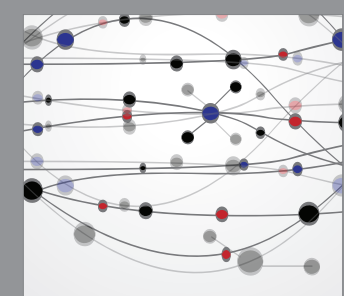

The Scientific World Journal
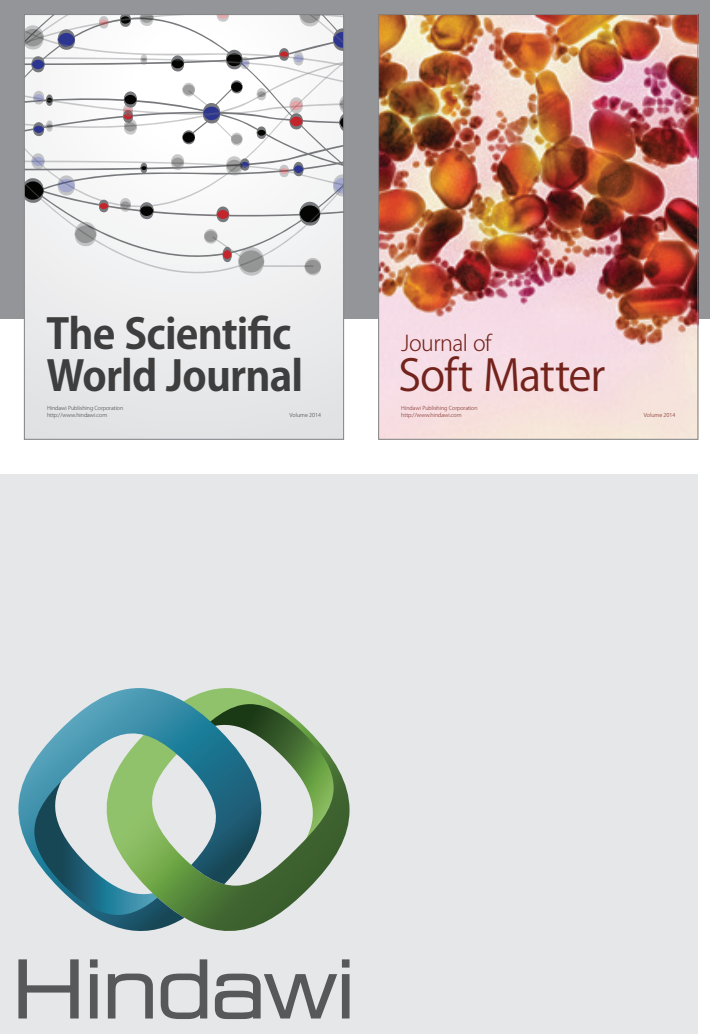

Submit your manuscripts at

http://www.hindawi.com
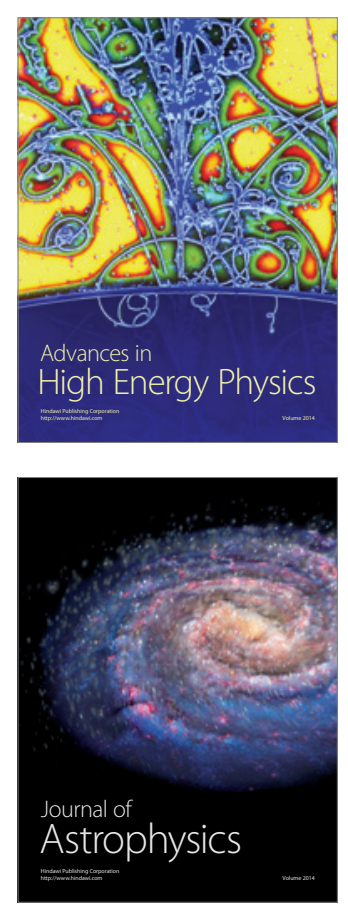
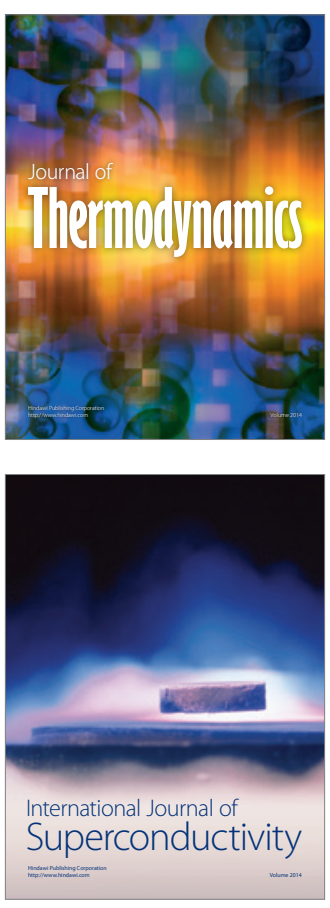
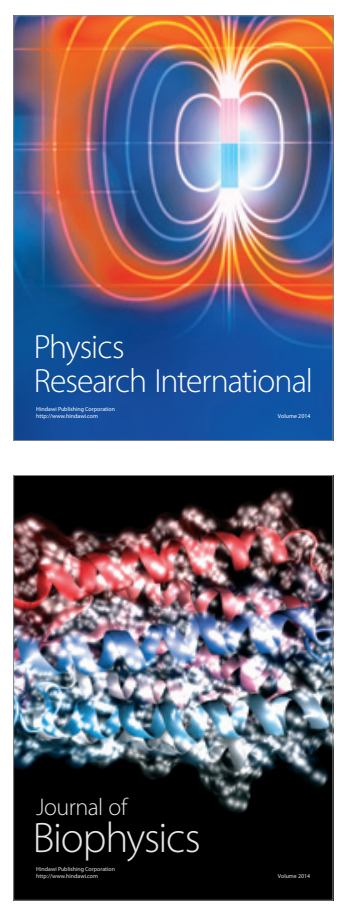
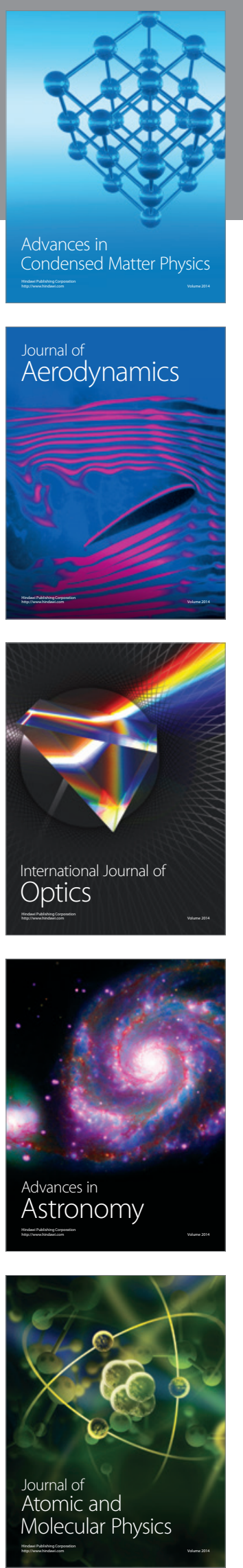